\title{
Geología para el estudio de microzonación sísmica en Santiago de los Caballeros, República Dominicana
}

\author{
M. Llorente Isidro ${ }^{(1)}$, M. Belvaux ${ }^{(3)}$, E. Bernardez ${ }^{(2)}$, D. Bertil ${ }^{(3)}, J$. A. Fernández Merodo ${ }^{(1)}$, L. Laín Huerta ${ }^{(1)}$, \\ E. Lopera Caballero(1), S. Muñoz Tapia ${ }^{(4)}$ y A. Roullé(3) \\ (1) Instituto Geológico y Minero de España, C/ Ríos Rosas 23; 28003 Madrid, España \\ m.llorente@igme.es; jose.fernandez@igme.es; I.lain@igme.es; e.lopera@igme.es \\ (2) Departamento de Geología, Universidad de Atacama. Avda. Copayapu 485, Copiapó, Atacama, Chile \\ enrique.bernardez@uda.cl \\ (3) Bureau de Recherches Géologiques et Minières; 3 avenue Claude-Guillemin - BP 3600945060 Orléans Cedex 2 - France \\ m.belvaux@brgm.fr; d.bertil@brgm.fr; a.roulle@brgm.fr \\ (4) Servicio Geológico Nacional. Av. Winston Churchill No. 75, Santo Domingo, República Dominicana \\ smunoz@sgn.gov.do
}

\begin{abstract}
RESUMEN
Tras el terremoto de Haití de 2010 dio comienzo una iniciativa para tratar de entender mejor los efectos de los terremotos en la República Dominicana, en particular en la ciudad de Santiago de los Caballeros, segunda más importante del país. Santiago ha sufrido varios terremotos devastadores, en 1562 la ciudad fue reconstruida en un nuevo sitio al sur de la falla responsable. El daño debido a un terremoto ocurre asociado a un conjunto de factores, entre ellos, la aceleración del suelo, que por lo general se señala como el principal o como la clave para explicar la mayor parte de los efectos. La aceleración del suelo varía dependiendo principalmente de la distancia al epicentro, de las propiedades del suelo y de la topografía. En cuanto a la distancia, es de esperar menor aceleración conforme aumenta la distancia al epicentro. De otra parte, suelos con diferentes características dan lugar a diferentes respuestas. Los efectos de la topografía aún no se comprenden bien. El efecto de la atenuación de la distancia en un radio de unos pocos kilómetros suele ser mucho menos relevante que el efecto de la variación en las propiedades de los suelos. Este artículo recoge los resultados obtenidos de los estudios de peligro sísmico y microzonificación sísmica en la ciudad de Santiago de los Caballeros: i) cuantificación del peligro sísmico regional debido a la falla septentrional; ii) un nuevo mapa geológico y iii) la cartografía de zonas de respuesta sísmica homogénea y susceptibilidad a la licuefacción.
\end{abstract}

Palabras clave: efectos de sitio, geotecnia, licuefacción, peligro sísmico, sismos.

\section{Geology applied to the study of seismic microzoning of Santiago de los Caballeros, Dominican Republic}

\begin{abstract}
After the Haiti quake of 2010 an initiative started to better understand shaking effects in the Dominican Republic after natural earthquakes, in particular in the city of Santiago de los Caballeros, the second city in the country as far as inhabitants and economic wealth are concerned. Santiago has suffered several devastating earthquakes; in 1562 the city was rebuilt on a new site (the current location) further south from the responsible fault. It is well known that damage caused by an earthquake occurs associated to a number of factors, ground acceleration is one of them and is usually considered as the key to explain most of the damage. Ground acceleration varies from one point to another depending mainly on: distance to the source of the rupture, soil properties and topography. Regarding the distance, the further from the source the less acceleration is expected due to distance attenuation. On the other hand, different soil properties and different topographies will produce different responses to the propagating wave. Within the range of a few kilometres, the effect of distance attenuation might be far less relevant than the effect of the varying properties of soils. This paper gathers together results obtained from the seismic hazard and microzonation studies developed in the city of Santiago: i) quantification of regional seismic hazard dominated by the Septentrional fault, ii) a new geological mapping of superficial formations, and iii) mapping of zones of homogeneous seismic response and liquefaction susceptibility.
\end{abstract}


Llorente Isidro, M., et al., 2017. Geología para el estudio de microzonación sísmica en... Boletín Geológico y Minero, 128 (3): $715-736$

Keywords: seismic hazard, earthquakes, geotecnics, site effects, liquefaction.

\section{ABRIDGED ENGLISH VERSION}

Hispaniola Island is located between the Caribbean and the North American tectonic plates where seismic activity can be very intense as demonstrated by the devastating quake of 2010 in Haiti. In order to highlight sensitive sectors where considering seismic risk must be given priority, it was decided to carry out a microzonation study in Santiago de los Caballeros, the second city in the Dominican Republic and particularly exposed because of its close location to a set of major active faults known as the Septentrional Fault Zone (SFZ). This study was funded by ONFED, supervised by the Dirección General de Mineria, coordinated by a technical unit, and executed by the IGME-BRGM-INYPSA consortium.

The oblique $20 \mathrm{~mm} / \mathrm{yr}$ convergence between the two plates (DeMets et al., 2000) is partitioned between subduction zones of the northern and south-eastern coasts of Hispaniola and strike-slip faults that transect the northern and southern portions of the island. The SFZ in the north of the island with ESE-WNW direction, accommodates part of this relative displacement (about $8 \pm 5 \mathrm{~mm} / \mathrm{yr}$, Manaker et al., 2008), that can therefore generate significant seismic hazard in the Santiago region. In order to provide a homogeneous seismicity catalogue, nearly 2,400 seismic events including historical ones have been collected within $200 \mathrm{~km}$ radius area covering the Santiago region. Magnitudes range from 3.5 to 8.1. This seismicity catalogue along with active fault database was used to establish a seismotectonic zonation in 13 seismic sources (Bertil et al., 2010). A probabilistic seismic hazard assessment was then carried out, estimating a peak ground acceleration (PGA) range for a 475 return period (10\% exceedance in 50 years) from $35 \%$ g to $41 \%$ g in Santiago de los Caballeros. The regional seismic hazard is clearly dominated by the Septentrional fault.

However, it is well known that the local geological conditions may change the response of a site to seismic excitation. Therefore, a geological campaign was carried out to better understand the soil characteristics in Santiago de los Caballeros, by means of a geotechnical study, outcrop observations and geophysical measures. In general terms, the superficial formations are mainly clay. Provided with such information, the city was divided into 7 units having similar geotechnical and geological properties and therefore similar seismic effects could be foreseen. 1D numerical modelling of the representative soil columns provided five site-specific response spectra. However, the results were not interpretable in two zones among the 7 units due to the complex geological and geometrical setting (strong lateral variations in velocities in contradiction with the $1 D$ hypothesis; succession of lithological formations without strong impedance contrast).

Following the site effects study, a qualitative analysis of the soil properties provided three susceptibility classes for mapping soil liquefaction susceptibility.

\section{Introducción}

La isla de La Española, compartida por la República Dominicana y Haití, está ubicada en la zona del límite convergente de la placa caribeña con la norteamericana y presenta una importante actividad tectónica (Figura 1). Fruto de dicha actividad fue el terremoto de Haití del 12 de enero de 2010, que ocasionó un gran desastre del que el país aún no ha logrado recuperarse. Como consecuencia de este evento, se movilizó ampliamente la opinión pública y en la República Dominicana, en 2010, se tomó la decisión de realizar un estudio de microzonificación sísmica de la ciudad de Santiago de Los Caballeros. Se escogió la ciudad de Santiago de los Caballeros por ser la segunda más importante del país después de Santo Domingo, la capital, en términos económicos y de población, y estar situada en una zona de fallas activas denominada "Zona de Falla Septentrional" (Figura 1). En el pasado, la ciudad se ha visto afectada por seísmos muy significativos, desde el más antiguo conocido en 1562 hasta el más reciente en Puerto Plata en 2003. Los estudios de microzonificación sísmica persiguen la caracterización de una región en relación con la respuesta al efecto sísmico por parte de los materiales geológicos en superficie. Fundamentalmente se trata de identificar áreas de similar amplificación o atenuación sísmica, como principal exponente de la peligrosidad sísmica y herramienta imprescindible para una adecuada gestión del riesgo sísmico a escala local. Este estudio se realizó en el marco de los proyectos de cartografía geotemática desarrollados en los programas SYSMIN I y SYSMIN II, financiados por el Fondo Europeo de Desarrollo con la Oficina Nacional para los Fondos Europeos (ONFED) como autoridad contratante, con la Supervisión de la Dirección General de Minería, la Coordinación por parte de una Unidad Técnica de Gestión (UTG), y con los socios IGME, BRGM e INYPSA.

El estudio del peligro sísmico se puede abordar 
desde diferentes perspectivas. Por un lado, el peligro sísmico regional persigue la caracterización de una región en términos de zonas sismo-tectónicas similares; es decir, que existan agrupaciones de observaciones sísmicas que se puedan asociar a procesos tectónicos similares, en un mismo régimen de esfuerzos en una misma zona de estructuras geológicas afines. Con ello, se puede evaluar la probabilidad de ocurrencia de terremotos que compartan su génesis y su asociación a las estructuras geológicas y al estado tensional del territorio, y por tanto, se puede conocer la relación entre la magnitud, la posición y la probabilidad de ocurrencia de distintos eventos teóricos, o su transformación a aceleraciones pico del suelo si éste fuera rocoso y homogéneo. Esa transformación conforma el mapa de peligrosidad sísmico regional y refleja la atenuación de la aceleración del suelo por el efecto de la distancia. El uso de este tipo de cartografías suele estar restringido a normativas de carácter general, como son las normas de construcción sismoresistentes.

Otra forma de abordar la peligrosidad sísmica es evaluar la respuesta del suelo considerando las condiciones locales o de sitio (lo que se conoce como la evaluación del peligro sísmico local), y que vienen determinadas por la composición de los materiales geológicos, su estructura y agrupación, su estado de consolidación, geometría, etc. Aún otra forma de abordar el peligro sísmico consiste en zonificar el territorio desde el punto de vista de otros fenómenos que serían desencadenados por un terremoto, como por ejemplo, movimientos del terreno, agrietamientos o licuefacciones, entre otros.

En este estudio, se realizó una zonificación sísmica regional, que sirvió para alimentar el modelo de amplificación sísmica por efecto de sitio, se hizo una microzonación sísmica que consiste en cartografiar las zonas de respuesta sísmica homogéneas $y$, finalmente, debido a la naturaleza de los materiales geológicos, se evaluó su probabilidad relativa a manifestar procesos de licuefacción.

\section{Contexto geodinámico}

Para comprender bien el contexto geodinámico imperante, es importante entender las relaciones entre las placas de Cocos, Nazca, Sudamérica y, sobre todo, Norteamericana con Caribeña, entre cuyo límite se encuentra la Isla de La Española. La convergencia de las placas caribeña y norteamericana es oblicua de unos $20 \mathrm{~mm}$ /año (DeMets et al., 2000), con un contexto general transpresivo, habiendo zonas de subducción al norte y sur de la isla, mientras que en su inte- rior predominan los desgarres transpresivos (Mann et al., 1984; Dolan et al., 1998; Manaker et al., 2008). En la actualidad dos de las zonas de falla transpresiva tienen una importante actividad tectónica, la Falla de Enriquillo que ha causado el reciente terremoto de Haití (12/01/2010) y la Zona de Falla Septentrional, cuya traza superficial pasa a escasos kilómetros de la ciudad de Santiago de los Caballeros.

El límite entre las placas Caribeña y Norteamericana en la región de La Española es una zona de deformación compleja (Mann et al., 1984). En particular, pasa de un régimen de desgarre senestro de dirección $N 75^{\circ} \mathrm{E}$ al Oeste (fallas de Islas Cisne, Oriental y Enriquillo-Plantain Garden), a una estructura transpresiva de dirección N95 ${ }^{\circ} \mathrm{E}$ hacia el Este (subducción de Muertos y de Puerto Rico, Figura 1). Esta zona de transición está dividida entre las zonas de subducción de las costas norte y sudeste de La Española y las fallas direcciónales que atraviesan las porciones norte y sur de la isla. Como consecuencia, el desplazamiento relativo de placas es acomodado en estructuras tectónicas de diferente naturaleza: fallas direcciónales senestrales de La Española hacia el oeste (zonas de falla de Islas Cisne, Oriente, Enriquillo-Plantain Garden y Septentrional); y la zona de subducción de La Española hacia el este (fosas Muertos y Puerto-Rico).

La Zona de Falla Septentrional (ZFS, Figura 1), recorre la parte norte de la isla con una dirección ESEONO. El movimiento de la falla es transpresional senestro con un desplazamiento de unos $8 \pm 5$ $\mathrm{mm} / \mathrm{año} \mathrm{de} \mathrm{acuerdo} \mathrm{con} \mathrm{Manaker} \mathrm{et} \mathrm{al.} \mathrm{(2008),} \mathrm{resal-}$ tando la incertidumbre en la cuantificación del desplazamiento. El desplazamiento es coherente, al

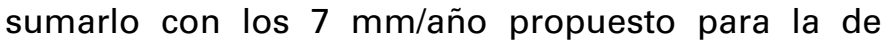
Enriquillo, con el valor global de $20 \mathrm{~mm} /$ año determinado para la placa caribeña por De Mets et al. (2000).

Las dos regiones o dominios tectonoestratigráficos separados por la falla tienen caracteres muy distintos. Al Norte, la cordillera septentrional, con importantes relieves, presenta una estructura geológica compleja, con materiales de edades muy variables, desde Cretácico Inferior a Plioceno, frecuentemente afectados por fuertes buzamientos, y con una tectónica transpresiva, con fallas que delimitan bloques con fuertes desplazamientos. Al Sur, el Valle del Cibao, por el contrario, se caracteriza por un relieve muy suave, prácticamente plano y una estratigrafía y tectónica mucho más sencillas, con relleno exclusivamente neógeno, que presenta buzamientos muy bajos y tectónica frágil, con desplazamientos mucho menos importantes que los de la Cordillera Septentrional. 


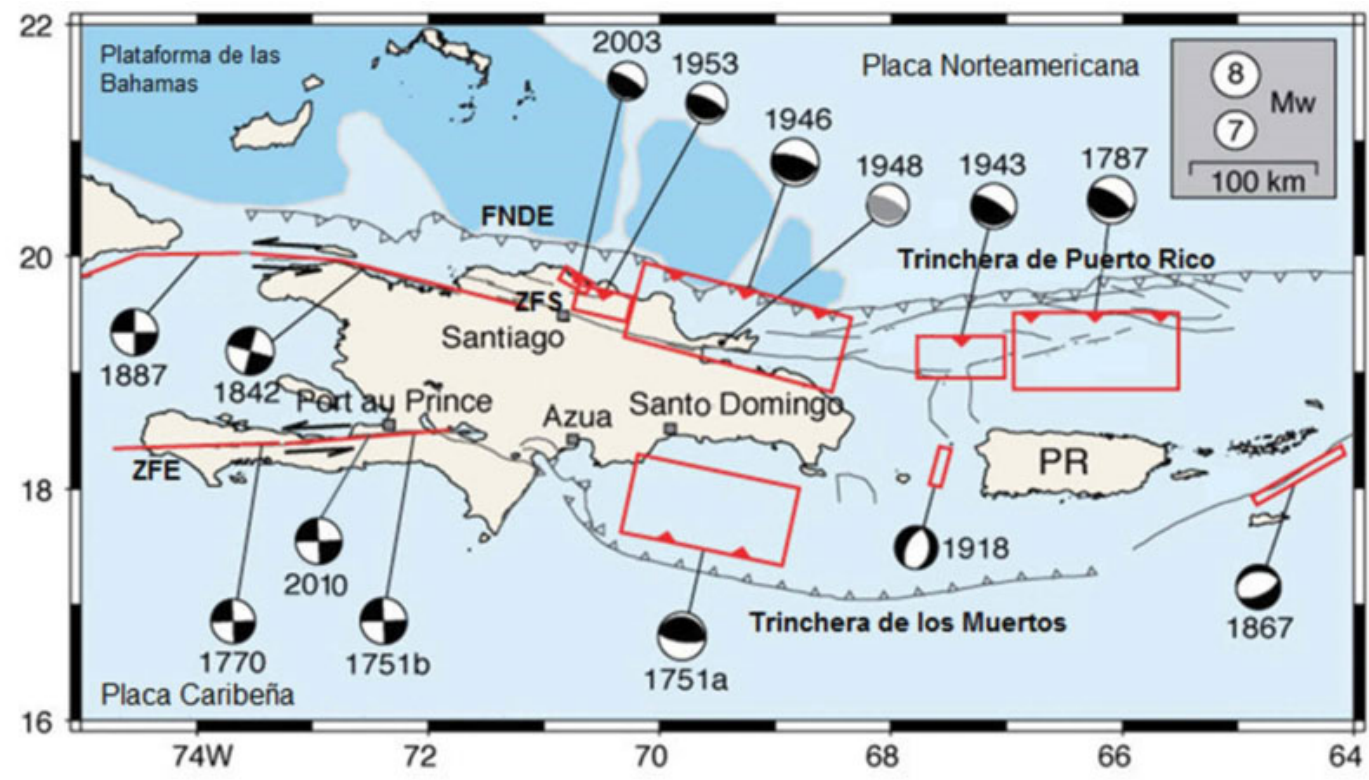

Figura 1. Localización de los principales accidentes tectónicos y mecanismos focales para algunos de los principales terremotos históricos. FNDE: Frente Norte de Deformación de la Española; ZFE: Zona de Falla de Enriquillo; ZFS: Zona de Falla Septentrional; PR: Puerto Rico. Modificado de Ali et al. (2008).

Figure 1. Main tectonic features and focal mechanisms for some of the most important historical earthquakes. FNDE: north front of the Deformation of La Española. ZFE: Enriquillo Fault Zone; ZFS: Septentrional Fault Zone; PR: Puerto Rico. Modified from Ali et al. (2008).

\section{Contexto sísmico}

\section{Sismicidad histórica e instrumental}

La actividad tectónica ligada a la zona de Falla Septentrional en las proximidades de la ciudad de Santiago ha dado lugar a importantes terremotos en época prehistórica e histórica, recogidos en los catálogos MIDAS y NOAA. Estudios neotectónicos realizados mediante zanjas en la parte central de la falla por Prentice et al. (1993) y Mann et al. (1998) indican que la última ruptura importante tuvo lugar hace unos 800 años y desde entonces se han acumulado unos $5 \mathrm{~m}$ de deformación, siendo muy elevada la probabilidad de un gran seísmo. Una parte de la energía acumulada, probablemente muy pequeña, se liberó en terremotos históricos, entre otros el que dio lugar a la destrucción total de la antigua ciudad de Santiago de los Caballeros el 2 de diciembre de 1562. Por aquel entonces la ciudad se encontraba emplazada al norte de su posición actual en la zona de Jacagua, directamente sobre la Zona de Falla Septentrional y tras la destrucción fue trasladada a su posición actual, en el mismo lugar en que Cristóbal Colón había fundado una fortaleza en 1495. Aun así la ciudad sufrió otras destrucciones por terremotos en 1776 y 1783 . En el año 1842 tuvo lugar el terremoto de mayor intensidad asociado a la Zona de Falla Septentrional. Con una intensidad de VIII-IX provocó nuevamente la destrucción de Santiago y otras ciudades de la isla, además de un maremoto que ocasionó la inundación de Montecristi y Manzanillo (Kelleher, 1973; Dolan, 1998). Otro terremoto, con epicentro también en la Zona de Falla Septentrional, al norte de Navarrete, provocó la destrucción de gran parte de la ciudad en 1897 (Prentice et al., 1993).

Para este estudio se ha compilado un catálogo sísmico unificado (Bertil et al., 2010, Figura 2) basado en distintos catálogos (MIDAS, ISC, NEIC/USGS, NOAA, Engdahl y Villaseñor (2002)). Este catálogo sísmico integra tanto la sismicidad histórica como la instrumental, abarcando en total el periodo entre el año 1564 y el año 2010 , para una ventana entre los $75^{\circ}$ y los $68^{\circ} \mathrm{O}$ de longitud y los $17^{\circ}$ y los $21^{\circ} \mathrm{N}$ de latitud, lo que incluye la totalidad de la isla de La Española. EI catálogo contiene cerca de 2.400 eventos sísmicos, de los cuales casi 700 están asociados a una magnitud Mw convertida. La magnitud mínima del catálogo es 3,5 y la magnitud máxima es 8,1 . Muchos datos históricos en este catálogo no presentan estimaciones de magnitud, siendo principalmente réplicas de los mayores eventos. 


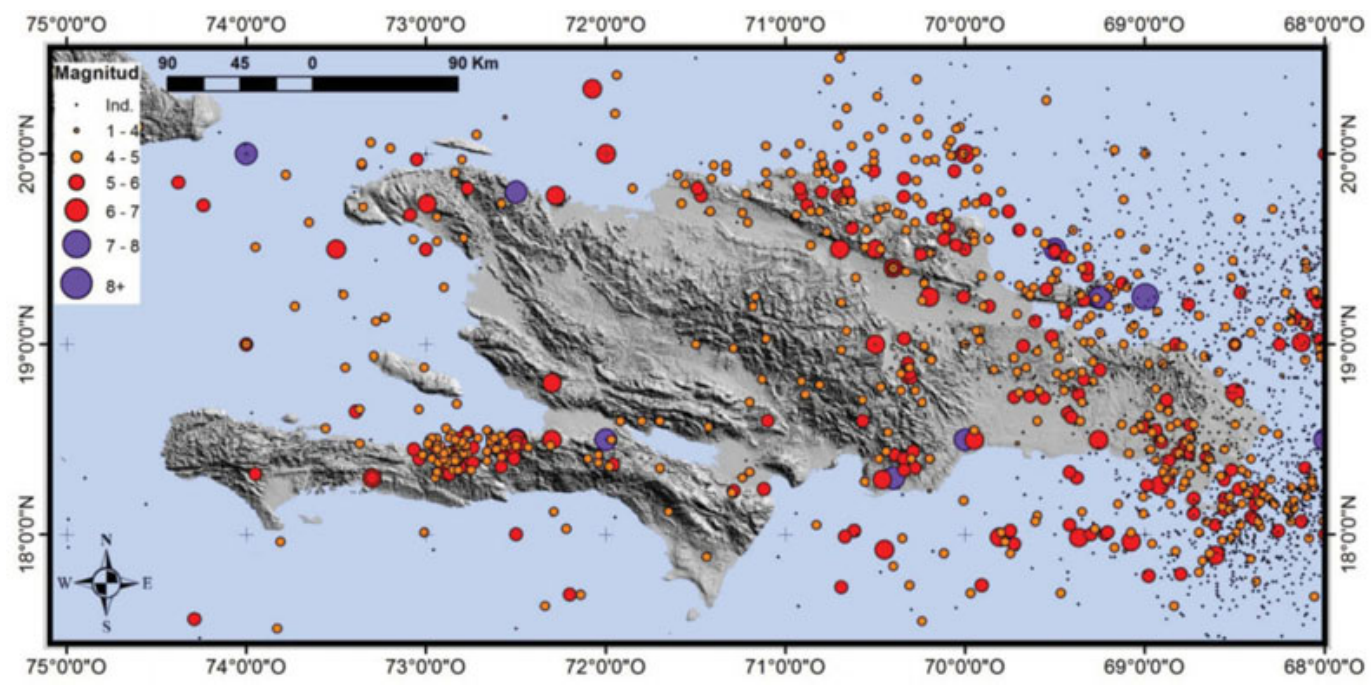

Figura 2. Epicentros de los sismos recolectados para el periodo 1564-2010 (basado en el catálogo unificado de Bertil et al., 2010). Figure 2. Epicentres from earthquakes collected from 1564 to 2010 (based on unified seismic catalogue from Bertil et al., 2010).

\section{Fuentes sismogénicas}

En una región tectónica activa como la isla de La Española, todas las observaciones se utilizaron para describir las fuentes sismogénicas y delimitar unidades o zonas sismotectónicas. Para la identificación de las fuentes sismogénicas potenciales, se tuvieron en cuenta los siguientes aspectos:

- Contexto geodinámico general.

- Situación geográfica

- Sismicidad asociada (histórica e instrumental)

- Grado de actividad tectónica, tipo de deformación y tasa de movimiento falla.

- Geología y geometría de la zona y del plano de

Las estructuras tectónicas que acomodan parte del desplazamiento relativo de las placas CaribeñaNorteamericana en la zona central de La Española e inmediaciones y que pueden generar un peligro sísmico significativo son:

- Zona de falla Enriquillo (o zona de falla EnriquilloPlantain Garden);

- Zona de falla Septentrional;

- Fosa de los Muertos;

- El sistema de fallas Norte-Española, es decir, la extensión occidental de la fosa de Puerto Rico al norte de La Española;

- En el seno de la isla de La Española, una zona de transición entre diferentes regímenes incluyendo la Cordillera Central.

Cada una de estas zonas de fallas activas ha sido responsable de grandes seísmos que han destruido varias poblaciones, entre las que se encuentra la ciudad de Santiago de Los Caballeros en diciembre de 1562. Las intensidades de los terremotos o de sus réplicas están en al rango de VII a IX y mayor (Kelleher et al., 1973; Dolan y Wald, 1998).

La zona de falla Enriquillo es una estructura E-O que atraviesa la parte sur de la isla de La Española (Mann et al., 1995). Esta zona acomoda desplazamientos siniestros paralelos al límite de placas, y presenta un plano de falla subvertical. Si bien la sismicidad instrumental ha sido relativamente baja (o desconocida por falta de una red sismológica), previamente a la secuencia de sismos de 2010. Del catálogo se pueden asociar a esta estructura entre cinco y seis sismos importantes ( $I_{0}$ de VIII o mayor).

La zona de falla Septentrional es una estructura de dirección E-O a ESE-ONO que atraviesa el norte de La Española, paralela al límite de placas. En el sector correspondiente a la República Dominicana, la zona de falla Septentrional se prolonga, más o menos segmentada, desde Montecristi hasta el sur de la península de Samaná formando el límite estructural entre la Cordillera Septentrional y el valle del Cibao. La sismicidad instrumental es baja a lo largo de la parte occidental de la zona de falla y más pronunciada a lo largo de la parte oriental. Se han identificado al menos tres eventos de $\mathrm{I}_{0}>\mathrm{VIII}$.

Para este trabajo la traza de la falla Septentrional ha sido cartografiada a partir del modelo de Mann et al. (2002), los datos SRTM30_PLUS topográficos y batimétricos (Becker y Sandwell, 2006) y las imágenes satélite LANDSAT. Se han identificado dos ramifi- 
Llorente Isidro, M., et al., 2017. Geología para el estudio de microzonación sísmica en... Boletín Geológico y Minero, 128 (3): $715-736$

caciones que se conectan al norte de la República Dominicana: la rama este (parte oriental de la zona de falla Septentrional) tiene una longitud de $820 \pm 80 \mathrm{~km}$ y la rama occidental (parte occidental de la zona de falla Septentrional) tiene una longitud cartográfica de $395 \pm 40 \mathrm{~km}$. Utilizando las relaciones de Wells y Coppersmith (1994), la zona de falla alcanza una magnitud máxima en torno a 8,3 $\pm 0,3$ y 7,9 $\pm 0,3 \mathrm{Mw}$ para los segmentos oriental y occidental de la falla. Frankel et al. (2010) asumen una longitud menor para estos segmentos y utilizan una magnitud máxima de 7,8 Mw para ambos segmentos.

La fosa de Muertos está orientada aproximadamente E-O, entre el estrecho Anegada al este y la cuenca de San Pedro al oeste (sur de La Española). La fosa acomoda fundamentalmente el acortamiento entre la placa Caribeña y el microbloque EspañolaPuerto Rico. La sismicidad instrumental asociada a esta estructura es moderada, y es difícil precisar la geometría. Sin embargo, es posible que esta zona generase un gran terremoto en 1751 (M 8 estimada) y produjese un tsunami (McCann, 2006). Se eligió el evento de M8 de 1751 como el terremoto potencial máximo en esta zona.

La zona intraplaca de transición de la cordillera viene marcada por la falla inversa de Matheux Neiba incluida en el trabajo de Frankel (2010). Es una de las fallas inversas que yacen bajo las montañas en Haití (Mann et al., 1995). Se incluye en la zona de transición "intraplaca" de la región de la Cordillera donde su geometría no se conoce bien (Maneker et al., 2008). La sismicidad registrada es difusa y superficial, y si bien parece que en 1911 se produjo un seísmo de $M w=7$, el tiempo de recurrencia para grandes terremotos en esta zona es más largo que en las fallas Enriquillo o Septentrional.

El sistema zona de falla Norte Española-subducción Puerto Rico señala el límite entre los microbloques (Gonave y La Española-Puerto Rico) y la placa Norteamericana. Se extiende desde la zona marina al noreste de La Española, donde conecta con la zona de subducción de Puerto Rico, a las costas sureste de Cuba donde es difícil de trazarla. Dentro del patrón actual de esfuerzos, la falla Norte Española acomoda una parte significativa de la convergencia entre La Española y la placa Norteamericana mientras que la componente lateral se acomoda en su mayoría por la zona de falla Septentrional. Aunque la intensa sismicidad instrumental en la zona de falla Norte Española puede ser atribuida principalmente al sector oriental de la estructura, esta actividad sísmica parece decrecer fuertemente hacia el oeste, si bien, la ausencia de una red sísmica en Haití no permite corroborarlo.

La región de estudio incluye también algunas uni- dades sismotectónicas poco activas y asociadas a una sismicidad moderada.

\section{Zonación sismotectónica}

Finalmente, en esta región tectónicamente compleja de límite de placas, se ha construido un modelo de 13 fuentes sismotectónicas (Bertil et al., 2010; Figura 3 y Tabla 1). Se han definido dos tipos de zonaciones: la primera distingue las zonas sismogénicas superficiales. En la segunda, la geometría de fuentes sísmicas tiene en cuenta las variaciones de profundidad asociadas a la subducción al Norte de la isla y en la zona de Los Muertos.

En este modelo, las fallas de Enriquillo y Septentrional se han tenido en cuenta individualmente en la zonación, ya que para estas fallas superficiales principales a las que se asocian seísmos históricos importantes, se dispone de datos de tasa de desplazamiento, las cuales permiten calcular los parámetros de actividad sísmica según la metodología descrita por Anderson y Luco (1983). Esta metodología aplica una zona de margen en el entorno de las fallas principales, donde se presupone la existencia de fallas anexas más pequeñas, en las cuales pueden generarse seísmos de magnitud baja a moderada.

Las otras fallas activas intraplacas superficiales de La Española son menos conocidas y se engloban en amplias zonas sísmicas poligonales, donde la sismicidad observada es más difusa y ahí los parámetros de actividad sísmica se calculan a partir de catálogos de sismicidad por aproximaciones estadísticas del tipo Weichert (1980).

LaTabla 1 resume las magnitudes máximas según cada zona sísmica (13 zonas cuyos números se reflejan en la Figura 3). La tercera y cuarta columnas indican las magnitudes máximas observadas a partir del catálogo de sismicidad y las magnitudes introducidas en el cálculo de amenaza probabilística.

\section{El peligro sísmico probabilístico}

Evaluar el peligro sísmico desde una perspectiva probabilística consiste en calcular la tasa anual de excedencia de una intensidad (o aceleración) en un lugar concreto. En la aproximación desarrollada por Cornell (1968), la actividad sísmica se supone aleatoria en el tiempo y en el espacio, y homogénea dentro de cada zona sismogénica. Este cálculo se ha realizado con el programa Crisis v. 2003. En cuanto a las relaciones de atenuación, y debido a la ausencia de una relación específica, se utilizaron las mismas relaciones que 

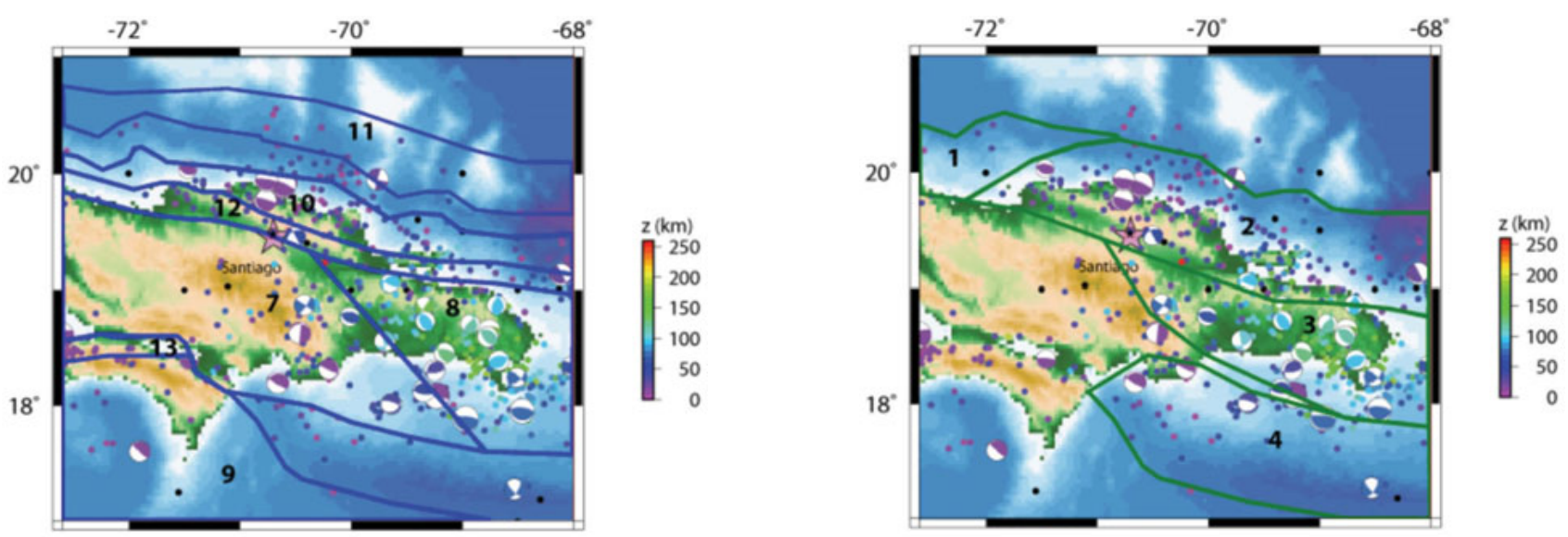

Figura 3. Modelo de zonación en 13 fuentes sísmicas; zonación en superficie a la izquierda, zonación en profundidad a la derecha. La sismicidad recopilada (Figura 2) está representada en función de la profundidad. Los mecanismos focales se toman del catálogo Harvard CMT: 1976-2010 (http://www.globalcmt.org/CMTsearch.html).

Figure 3. Zonation model in 13 seismic sources; surface zonation on the left, depth zonation on the right. The collected seismicity (Fig. 2) is plotted as function of depth. Focal mechanisms are taken from Harvard CMT catalog: 1976-2010 (http://www.globalcmt.org/CMTsearch.html).

\begin{tabular}{|c|c|c|c|}
\hline $\mathbf{N}$. & Nombre & $\begin{array}{c}\text { Mw máxima observada } \\
\text { (año correspondiente) }\end{array}$ & $\begin{array}{c}\text { Mw máxima e } \\
\text { incertidumbre }\end{array}$ \\
\hline 1 & Subducción Norte interfase Oeste & $6,6(1962)$ & $8,0 \pm 0,2$ \\
\hline 2 & Subducción Norte interfase Este & $8,1(1946)$ & $8,3 \pm 0,3$ \\
\hline 3 & Lámina Subductiva Norte & $7,0(1946)$ & $7,5 \pm 0,5$ \\
\hline 4 & Cabalgamiento de Muertos & $8,0(1751)$ & $8,2 \pm 0,25$ \\
\hline 5 & Background Septentrional & 5,5 & \\
\hline 6 & Background Enriquillo & 5,5 & $7,5 \pm 0,2$ \\
\hline 7 & Transición Cordillera & $7,0(1911)$ & $7,0 \pm 0,5$ \\
\hline 8 & Intraplaca Este Española & $5,5(1989)$ & $6,05 \pm 0,25$ \\
\hline 9 & Background Sur Haití Muertos & $5,8(1939)$ & $6,65 \pm 0,25$ \\
\hline 10 & Prisma de acreción Norte & $6,4(2003)$ & $6,0 \pm 0,5$ \\
\hline 11 & Convexidad externa & $4,9(1987-2003)$ & $8,15 \pm 0,15$ \\
\hline 12 & Zona de falla Septentrional & $8,0(1842)$ & $7,8 \pm 0,3$ \\
\hline 13 & Zona de falla Enriquillo & $7,5(1751-1770)$ & \\
\hline
\end{tabular}

Tabla 1. Unidades o zonas sismotectónicas y sismogénicas.

Table 1. Sismotectonic and sismogenic units or zones.

fueron utilizadas por Frankel et al. (2010), quienes evaluaron la amenaza sísmica de Haití tras el sismo del 12 de enero de 2010. Para ello utilizaron un juego de 6 relaciones de atenuación: 3 para las fallas corticales y 3 para la subducción. También fijaron una ponderación asociada a cada una de ellas para la consideración de los errores epistémicos ligados a esta selección de relaciones:

Para las fallas y las zonas fuentes de terremotos superficiales. Estas relaciones son:

- Boore \& Atkinson (2008); peso 1/3.

- Campbell \& Bozorgnia (2008), peso 1/3.

- Chiou \& Youngs (2008), peso 1/3.
Para los contactos en interfase de las zonas de subducción:

- Zhao et al. (2006), peso $1 / 2$.

- Youngs et al. (1997), peso $1 / 4$.

- Atkinson \& Boore (2003), peso 1/4.

El cálculo de la amenaza se efectúa en una cuadrícula de 35 puntos en la ciudad de Santiago cubriendo una superficie de $112 \mathrm{~km}^{2}$. El periodo de retorno es de 475 años ( $10 \%$ de probabilidad en 50 años). El resultado se puede observar en el mapa de aceleración pico del suelo (PGA) de la Figura 4, donde se aprecia que el peligro sísmico aumenta progresivamente del $\mathrm{SO}$ al NE de la ciudad (mínimo 0,33g, máximo $0,44 \mathrm{~g}$ ). 
El gradiente es perpendicular a la dirección de la falla Septentrional, mostrando así la fuerte influencia de esta fuente sísmica sobre el peligro sísmico. Por ese motivo se han seleccionado dos puntos de referencia, para representar el espectro de amenaza uniforme en los sectores de peligro más y menos elevados de la ciudad, uno al $\mathrm{SO}$ de la ciudad y otro en el $\mathrm{NE}$, que corresponden a valores de PGA de $0,35 \mathrm{~g}$ y $0,42 \mathrm{~g}$, respectivamente.

\section{Geología y geotécnia del área de estudio}

Para poder realizar un estudio de microzonificación sísmica es necesario contar con una adecuada información de la geología de la zona, lo que incluye la caracterización de unidades geológicas, formaciones superficiales y unidades lito-geotécnicas, así como su relación con las unidades circundantes. El marco de estudio es un rectángulo de unos $133 \mathrm{~km}^{2}$ (Figura 5), que en su mayor parte corresponde a áreas urbanas y una pequeña parte a terrenos de cultivo en terrazas fluviales. Los afloramientos en este perímetro son muy escasos, por lo que la cartografía geológica de detalle (escala 1/10.000) se realizó basándose en observaciones de obras y zanjas de carácter temporal, en la campaña de sondeos realizada, y en otros documentos recopilados (Figura 5 a Figura 9).

La cartografía geológica y lito-geotécnica muestra, a grandes rasgos, tres grandes grupos (Figura 6): los materiales pertenecientes a la Cordillera Septentrional; los neógenos del Valle del Cibao y los cuaternarios recientes.

\section{Cordillera Septentrional}

En el área de estudio, tan sólo aparece en una pequeña superficie (de unos $0,3 \mathrm{~km}^{2}$ ) de la esquina NE, perteneciendo todo ello al miembro La Pocilguita de la

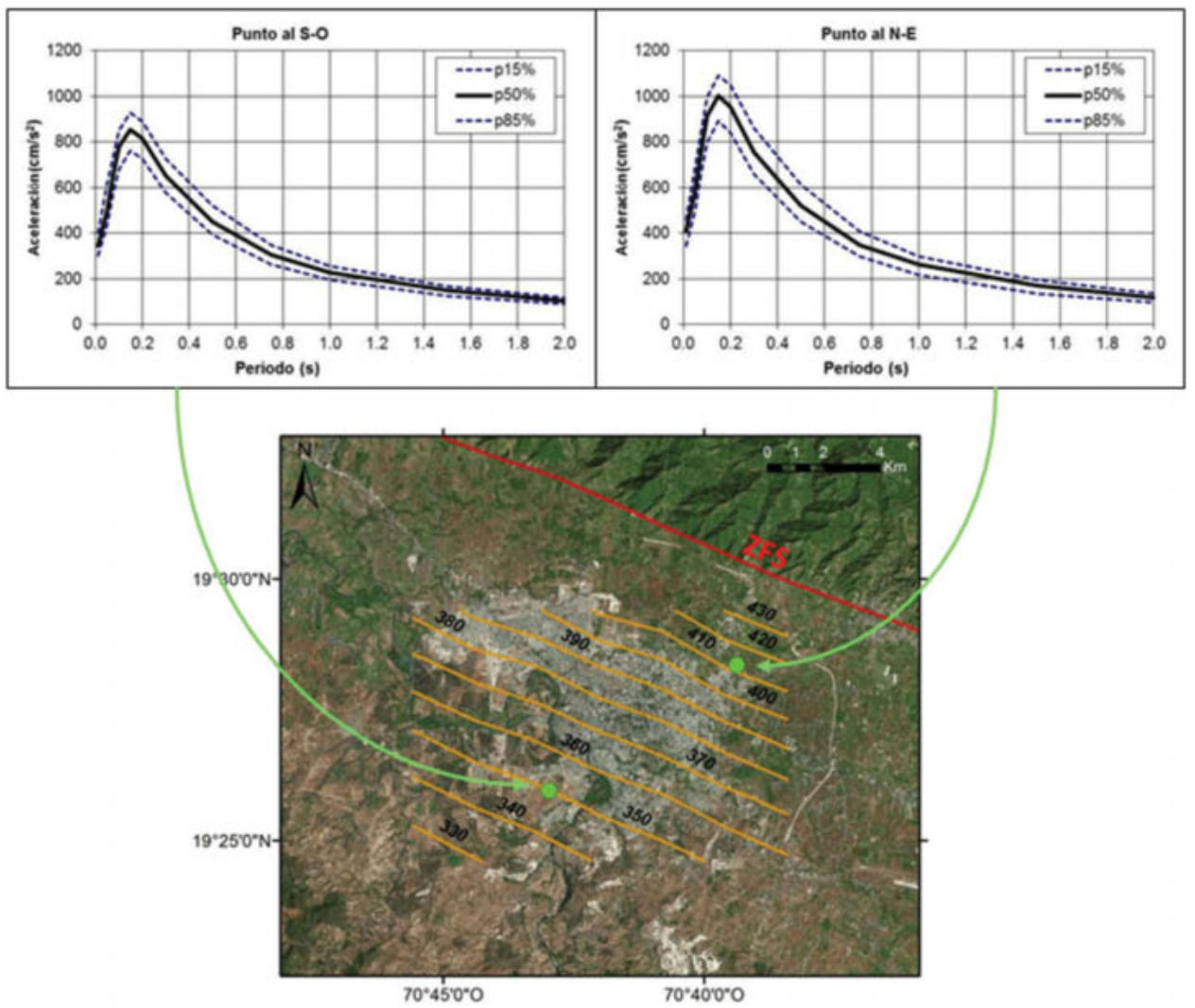

Figura 4. Espectros de peligro sísmico, puntos de cálculo y mapa de isolineas de aceleración pico del suelo (PGA, de 330 a $430 \mathrm{~cm} / \mathrm{s}^{2} \mathrm{SO}-$ NE con equidistancia $10 \mathrm{~cm} / \mathrm{s}^{2}$ ) de la ciudad de Santiago de los Caballeros paraT 475 años. Los espectros son representados para las aceleraciones medias y los percentiles $15 \%$ y $85 \%$. La línea roja indica el trazado de la ZFS.

Figure 4. Uniform hazard spectra, calculation sites and peak ground acceleration (PGA) isolines (from 330 to $430 \mathrm{SW}-\mathrm{NE}$ at $10 \mathrm{~cm} / \mathrm{s}^{2}$ equidistant) for Santiago de los Caballeros with T475 years. Spectra are represented for the mean PGA and the percentiles $15 \%$ and $85 \%$. The red line indicates the ZFS segment trace. 
Unidad de Altamira - La Toca. Está constituida principalmente por lutitas calcáreas (entre un 50 y $80 \%$ del total de la unidad) de color gris-marrón a beige, de grano medio a fino, y con cemento calcáreo, con gradación positiva y estructuras tractivas típicamente turbidíticas. Según Urien (2010), la edad de estos materiales es Mioceno y su potencia total se calcula en unos $300 \mathrm{~m}$.

\section{Neógeno y cuaternario marino del Valle del Cibao}

La mayor parte de la zona de estudio corresponde a los materiales neógenos y cuaternarios marinos del Valle del Cibao. La Cuenca del Cibao configura, de forma conjunta con la Cordillera Septentrional, una gran estructura sinclinal cuyo eje transcurre aproximadamente paralelo a la propia cuenca (Mann et al., 1991). La potencia estimada de la serie sedimentaria es de 5.000 a $6.000 \mathrm{~m}$, y está integrada por las Formaciones Bulla, Cercado, Gurabo y Mao (Mioceno inferior-Pleistoceno), de las cuales tan sólo afloran en la zona de estudio las formaciones Gurabo y Mao.

\section{Formación Gurabo}

La formación Gurabo está constituida por lutitas más o menos carbonatadas a las que Saunders et al. (1986) atribuyen una potencia de $425 \mathrm{~m}$. Hacia techo se interdigitan con las calizas de la Formación Mao Adentro, no representadas en el área de este estudio. La base de la Formación Gurabo se ha datado en el Mioceno superior, mientras que su techo se ha situado en el Piacenciense (Bernárdez, 2004). Dentro de los trabajos desarrollados en el proyecto SYSMIN, la Formación Gurabo se describe como una serie homogénea de margas azuladas que intercalan, en su parte superior, niveles de arenas y debris flows conglomeráticos seguidos de niveles de génesis similar, con fragmentos de corales y otros bioclastos. La Formación Gurabo muestra una secuencia de profundización hacia techo, que se inicia en un sistema paraarrecifal y finaliza en un medio de plataforma siliciclástica externa, por debajo de las Calizas de Mao Adentro (McNeill et al., 2008). La representación cartográfica de la Formación Gurabo ocupa aproximadamente un cuarto de la superficie de la hoja, situándose fundamentalmente en su tercio occidental. Los afloramientos ocupan generalmente depresiones: las riberas del Río Yaque en la zona urbana y periurbana de Santiago, así como los cortes antrópicos de carreteras, ofrecen las mejores secciones. En la zona de estudio se observaron lutitas variablemente calcáre- as, con alternancias de argilitas y limolitas, por lo general en capas decimétricas. En superficie los afloramientos presentan colores grisáceos claros, que se hacen amarillentos en las zonas más alteradas, donde suelen presentar venas irregulares de yesos fibrosos con potencias generalmente de unos pocos milímetros, raramente llegando al centímetro. En profundidad los colores son más oscuros, con tonalidades verdosas, llegándose en el caso de las argilitas más puras a colores casi negros. Estas coloraciones oscuras se deben a la presencia de abundante materia orgánica, en ambiente reductor, a la que suele acompañar pequeñas cantidades de pirita en forma de microcristales dispersos. La alteración de la pirita en presencia del cemento carbonatado da lugar en los afloramientos alterados a la presencia de venas de yeso fibroso, que pueden ser tanto paralelas como subperpendiculares a la estratificación. La macrofauna es generalmente escasa, con predominio de gasterópodos y bivalvos, que suelen presentarse fragmentados por aplastamiento. De acuerdo con Urien (2010) la potencia de la formación puede alcanzar los 200 metros en el marco de la Hoja de Santiago de Los Caballeros. El contacto con la Formación Cercado subyacente, no visible en el área estudiada, es una discordancia angular según Urien (2010). A techo, la Formación Gurabo, está en contacto con la Formación Mao (Piacenciense y Gelasiense), la Unidad de Licey al Medio (Pleistoceno post Gelasiense) y otros materiales cuaternarios. El contacto con la Formación Mao es una marcada discontinuidad.

\section{Formación Mao}

La base de la Formación Mao se había situado en el Zancliense a partir de las dataciones efectuadas en la parte alta de la Formación Gurabo (Saunders et al., 1986). Posteriormente, Bernárdez (2004) identifica y cartografía uno de los afloramientos de edad Piacenciense, atribuido previamente a la Formación Mao, como perteneciente a la Formación Gurabo. El techo corresponde siempre a la discordancia basal de los materiales cuaternarios. Se le concede una potencia mínima de 350-600 m y máxima de $3600 \mathrm{~m}$. En el área cartografiada las potencias son mucho menores, y no parece superar en mucho la centena de metros. En general, se puede asignar, con reservas, a su parte más alta, una edad pleistocena Gelasiense, con posibilidad de alcanzar el Pleistoceno Calabriense. La Formación Mao está compuesta por un conjunto de facies muy heterogéneas que reflejan un amplio abanico de medios sedimentarios. Erikson et al. (1998) 
describen la base de la Formación Mao como un contacto neto y erosivo, desarrollado sobre las margas y limos de la Formación Gurabo, que se encuentra tapizado por conglomerados y arenas de talud. Por otro lado, los trabajos realizados dentro del proyecto SYSMIN han demostrado que el límite entre ambas formaciones es complejo y de naturaleza muy variable según la zona de la cuenca considerada. La Formación Mao está constituida fundamentalmente por un conjunto siliciclástico heterogéneo, en que las litologías predominantes son areniscas, conglomerados y limolitas con algunas intercalaciones de arcillas y de limos calcáreos. Los limos suelen tener una participación arenosa importante y en ocasiones presentan fauna marina y costera, siendo más general la presencia de restos vegetales. Se han citado en esta unidad depósitos de debris flows y slumps lutíticos, entre los que se intercalan capas tabulares de areniscas laminadas con estratificación fina-media y conglomerados. La Formación Mao en general presenta características sedimentarias propias de medios deltaicos progradantes desde el Sur, principalmente en zonas próximas a su desembocadura, en cuencas con creación continua del espacio de acomodación y altas tasas de acumulación sedimentaria. En la cartografía realizada la Formación Mao ocupa fundamentalmente el tercio central de la hoja, definiendo su representación cartográfica una amplia banda de orientación NO-SE. En la hoja de Santiago (Urien, 2010) se ha dividido la Formación Mao en tres subunidades que se han reconocido en la presente cartografía: Areniscas con intercalaciones de lutitas beige y argilitas; Conglomerados y areniscas de grano medio en secuencias deltaicas; Unidad de Palo Amarillo, constituida por calizas con corales, brechas coralinas con intercalaciones de margas y cantos conglomeráticos, calizas bioclásticas, lumaquelas y conglomerado con matriz carbonatada.

\section{Cuaternarios recientes}

Además de los depósitos que se describen a continuación, durante el cuaternario más reciente se ha desarrollado una importante alteración de los materiales depositados previamente. Esta alteración, ligada principalmente a las oscilaciones del nivel freático provoca una oxidación de la materia orgánica y sulfuros contenidos en los sedimentos lutíticos, así como una disolución del cemento y los bioclastos carbonatados, siendo reconocible por el cambio de coloración, de grisáceo más o menos oscuro a pardo amarillento. Esta alteración provoca una importante modificación de las características litológicas y geo- técnicas de los materiales, su máxima profundidad ha sido representada en los cortes geológicos con una línea roja discontinua. La profundidad de este nivel oscila por lo general entre los 10 y 20 metros, pero en algunos puntos puede alcanzar los $34 \mathrm{~m}$ y en otros puntos, como el fondo de excavación de los cauces principales se sitúa en la superficie.

\section{Abanico aluvial}

Los afloramientos de abanicos aluviales se sitúan en el borde norte de la hoja, correspondiendo al piedemonte de la Cordillera Septentrional. Se interdigitan con la Unidad de Licey, constituyendo la parte proximal de la misma. Están constituidos por depósitos conglomeráticos con cantos de naturaleza fundamentalmente carbonatada y areniscas calcáreas procedentes de la Cordillera Septentrional.

\section{Unidad de Licey}

Ocupa aproximadamente un tercio de la superficie cartografiada, fundamentalmente en su mitad oriental. Morfológicamente se caracteriza por dar lugar a una Ilanura, con muy suave pendiente, dirigida casi uniformemente hacia el suroeste. La mayor parte de su superficie está ocupada por terrenos de cultivo, desarrollándose sobre ella suelos negros muy fértiles. Al norte se interdigita con la unidad de abanicos aluviales, y en superficie se dispone siempre sobre la formación Mao, si bien en sondeos se ha detectado sobre la Formación Gurabo y sobre las terrazas medias, con las que parece interdigitarse. Los afloramientos, muy escasos, se ubican en las incisiones naturales de los arroyos principales o en cimentaciones de edificios. La unidad presenta una fuerte anomalía de potasio que permite su teledetección (Urien, 2010). La litología que fundamentalmente constituye esta unidad son arcillas pardas masivas (con un contenido de esmectitas de entre el 23 y el $50 \%$, Urien, 2010) que cuando son excavadas por los arroyos tienen fuertes pendientes que frecuentemente presentan deslizamientos. Generalmente están saturadas en agua hasta un metro por encima del nivel de drenaje. Entre estas arcillas se intercalan niveles, de hasta un metro de potencia por lo general, de arcillas con arenas de diversa granulometría, llegando a veces a microconglomerados. Los cantos y granos están siempre flotando en la matriz arcillosa y nunca presentan ningún tipo de ordenación. 
Llorente Isidro, M., et al., 2017. Geología para el estudio de microzonación sísmica en... Boletín Geológico y Minero, 128 (3): $715-736$

\section{Conos de deyección}

Son pequeños abanicos aluviales que se sitúan en la desembocadura de pequeños arroyos. Los principales se sitúan en las proximidades del Yaque, sobre sus terrazas bajas y corresponden a tres arroyos que han quedado colgados por la excavación de la red actual. Su composición es arenosa-fangosa y la potencia del depósito es muy escasa, probablemente no superando los dos metros de máxima. Otro pequeño cono de este tipo se ha identificado por su expresión topográfica al oeste de los Cerros de Gurabo.

\section{Terrazas medias-altas}

Se ha agrupado en este epígrafe varios niveles de terrazas separados verticalmente del cauce actual cuya correlación por el momento resulta problemática. Así por ejemplo en la parte sur de la hoja se han identificado en la margen oriental del río Yaque tres niveles de terrazas, ninguno de los cuales es directamente correlacionable con los al menos dos niveles que se pueden identificar inmediatamente al oeste de dicho río. La litología más característica son los conglomerados de cantos rodados de tamaño variable flotantes en una matriz de arenas sueltas. La composición de los cantos es fundamentalmente de rocas ígneas y metamórficas procedentes de la cordillera central. Volumétricamente son más importantes los tramos de arenas y limos, aunque en superficie suelen encontrarse muy recubiertos y los afloramientos son muy escasos. En las proximidades de Cerro Alto hay afloramientos de estos niveles de limos con arenas finas que contienen huellas de raices y gasterópodos continentales. Presentan una estratificación ondulante generada por procesos de licuefacción.

\section{Terrazas bajas}

Las terrazas aluviales bajas están en relación directa con la red hidrográfica actual o la llanura de inundación. Sus afloramientos se sitúan en las proximidades del cauce del río Yaque. Estas terrazas están constituidas por cantos de tamaño centimétrico y decimétrico, polimícticos, de rocas intrusivas, metamórficas y volcánicas procedentes directa o indirectamente de la Cordillera Central. Ocasionalmente se encuentran cantos de calizas coralinas. La matriz de arenas gruesas no está consolidada. Estas terrazas pueden ser inundadas durante las grandes crecidas del Río Yaque como ocurrió durante la Tormenta Olga de diciembre
2007. En la actualidad el río Yaque circula encajado en estas terrazas, que llegan a una profundidad netamente mayor que la actual de excavación del cauce.

\section{Llanura de inundación y canales abandonados}

Las facies de llanura de inundación se concentran en las orillas de las partes anchas del curso del RíoYaque del Norte. Debido al régimen subtorrencial del río, los depósitos están constituidos fundamentalmente por cantos redondos de tamaño centimétrico a métrico y son clasto-soportados con muy poca matriz arenosa. El mayor desarrollo de estos depósitos se concentra aguas abajo del centro de Santiago, donde el río, después de dibujar un fuerte meandro, cambia la dirección Sur-Norte que tiene aguas arriba, a la Este-Oeste que mantiene hasta su desembocadura.

\section{Aluviones de fondo de valle}

Los aluviones de fondo de valle están presentes en los principales arroyos, estando constituidos en general por depósitos de energía moderada, fundamentalmente fangosos y arenosos de colores pardos oscuros, con abundante materia orgánica y algunas lentes conglomeráticas. Su potencia es por lo general muy reducida, de poco más de un par de metros. Los aluviones del río Yaque son de mayor energía y están constituidos por cantos redondeados de diámetro muy variable y con muy poca matriz arenosa en superficie. En la cartografía se han incluido con estos depósitos las barras laterales y centrales del río, con más participación de material arenoso.

\section{Aluvial-Coluvial}

Son depósitos que ocupan zonas de escaso relieve y parecen corresponder a un retrabajamiento de coluviones y suelos por escorrentía superficial difusa. Tienen una potencia muy escasa, por lo general menor de un metro y están constituidos principalmente por limos y arcillas con cantos dispersos y en los que no se aprecia ningún tipo de ordenación.

\section{Glacis aluvial}

Estos depósitos ocupan planicies inclinadas en zonas de cabecera de valles en la esquina suroccidental de la hoja. Están constituidos fundamentalmente por limos y arenas limosas con algunos horizontes de 
gravas. También son frecuentes los niveles con abundantes gasterópodos. Presentan una laminación paralela bastante marcada por lo general, con algunos horizontes de bioturbación de raíces. Su depósito podría corresponder a una escorrentía superficial con flujo laminar. Parecen rellenar fondos de valle posteriormente escavados por la red actual. Su potencia puede llegar a superar los $8 \mathrm{~m}$. Su superficie está ocupada por cultivos y se sitúan en zonas con muy escasa probabilidad de desarrollo urbano.

\section{Coluvión}

Estos depósitos gravitacionales de ladera se sitúan a pie de fuertes escarpes, situados fundamentalmente en la Formación Gurabo y se componen de cantos de dicha unidad en una matriz de fangos resultante de la descomposición de la misma. Tanto su potencia como su distribución areal son muy reducidas.

\section{Depósitos antrópicos}

Tratándose el área cartografiada de un área urbana los depósitos de origen antrópico son prácticamente omnipresentes, y en la cartografía se han representado únicamente los de mayor volumen o importancia cara a la evaluación de riesgos. Se han representado depósitos antrópicos de tres tipos; vertederos, escombreras y zahorras de apoyo de grandes construcciones. Los vertederos de Rafey o de Cienfuegos son los más importantes de la comunidad urbana de Santiago. Son acumulaciones de basuras de origen doméstico o industrial, depositadas en las canteras abandonadas de las lutitas de la Formación Gurabo o terrazas medias del Río Yaque. Otros vertederos, de mucha menos importancia volumétrica, pero significativos por estar parcialmente ocupados por viviendas, se han cartografiado por distintas partes de la ciudad, especialmente aquellos asentados sobre arciIlas de la Unidad de Licey con fuerte pendiente y desnivel, y que sirven de asiento a un número elevado de viviendas. Se han cartografiado otros vertederos de menor importancia pero con área cartografiable, generalmente a orillas de arroyos, pero los vertederos de dimensiones inferiores a los $10 \mathrm{~m}$ cuadrados son sumamente numerosos. Se han cartografiado dos acumulaciones de escombros, una en el emplazamiento del sondeo 10, próximo al campo de golf, y otra, especialmente significativa, en la parte occidental de la Avenida Hatuey en el Barrio de La Rosa, sobre la que hay varias edificaciones afectadas por grietas.

\section{Detalles de las campañas de geología y de geotecnia}

En concreto, se han empleado 214 sitios de observación entre afloramientos, zanjas y obras, con una muy buena distribución espacial; 18 sondeos geomecánicos con recuperación continua de testigo (con profundidades de entre 18 y $70 \mathrm{~m}$; unos $640 \mathrm{~m}$ totales) y 55 sondeos recopilados (con profundidades de entre 2 y $27 \mathrm{~m}$; unos $360 \mathrm{~m}$ totales). Como antecedentes de apoyo a esta tarea se contaba con los trabajos previos del proyecto SYSMIN (Urien, 2010) a escala $1 / 50.000$, la cartografía 1/13.000 de Franco y Peña (2003), la recopilación de sondeos de Penson (1973) y otros documentos recopilados sobre el terreno de trabajos geotécnicos previos. Adicionalmente se realizó el mapa topográfico con callejero a escala 1/10.000 a partir de fotorrestitución de pares estereoscópicos aéreos verticales de la ciudad. De la campaña geotécnica se derivaron un total de 461 ensayos de laboratorio y 344 ensayos in situ. Los ensayos de laboratorio han sido granulometrías (88), límites de Atterberg (88), contenido de humedad natural (83), clasificación de suelos (88), compresión uniaxial simple (21) y densidad (93). El tipo de ensayo in situ ha sido el Standard Penetration Test (SPT, 344) cada $1,5 \mathrm{~m}$ ó en profundidades o dificultades locales singulares cada $3 \mathrm{~m}$ o menos. La campaña geofísica también aportó información relevante para la elaboración del mapa geológico y geotécnico. Se hicieron 57 medidas SASW (Spectral Analysis of Surface Waves) y $150 \mathrm{H} / \mathrm{V}$ (ruido de fondo).

Los suelos de Santiago presentan un peso específico de 1,79 a $1,14 \mathrm{gr} / \mathrm{cm}^{3}$ y una humedad natural del 35 al $10 \%$. En cuanto a los límites de Atterberg para los casos estudiados, el límite líquido es el que más variabilidad presenta, con un máximo de 66 y un mínimo de 10; mientras que el límite plástico se mantiene bastante constante entre 17 y 25 . El índice de plasticidad indica que predominan los materiales muy plásticos, destacando algunas muestras con valores muy elevados (máximo $I P=43$ ). Además, sobre el terreno se ha podido comprobar que algunos niveles son expansivos, especialmente las arcillas de Licey (en los sondeos 13 y 14 se han observado hinchamientos superiores al $20 \%$ en algunos tramos; IGME, 2010).

\section{Microzonificación sísmica}

Las condiciones particulares de un lugar (naturaleza y potencia de las formaciones superficiales, distancia en relación a la fuente sísmica) pueden cambiar radicalmente el potencial destructivo de un sismo, inclu- 


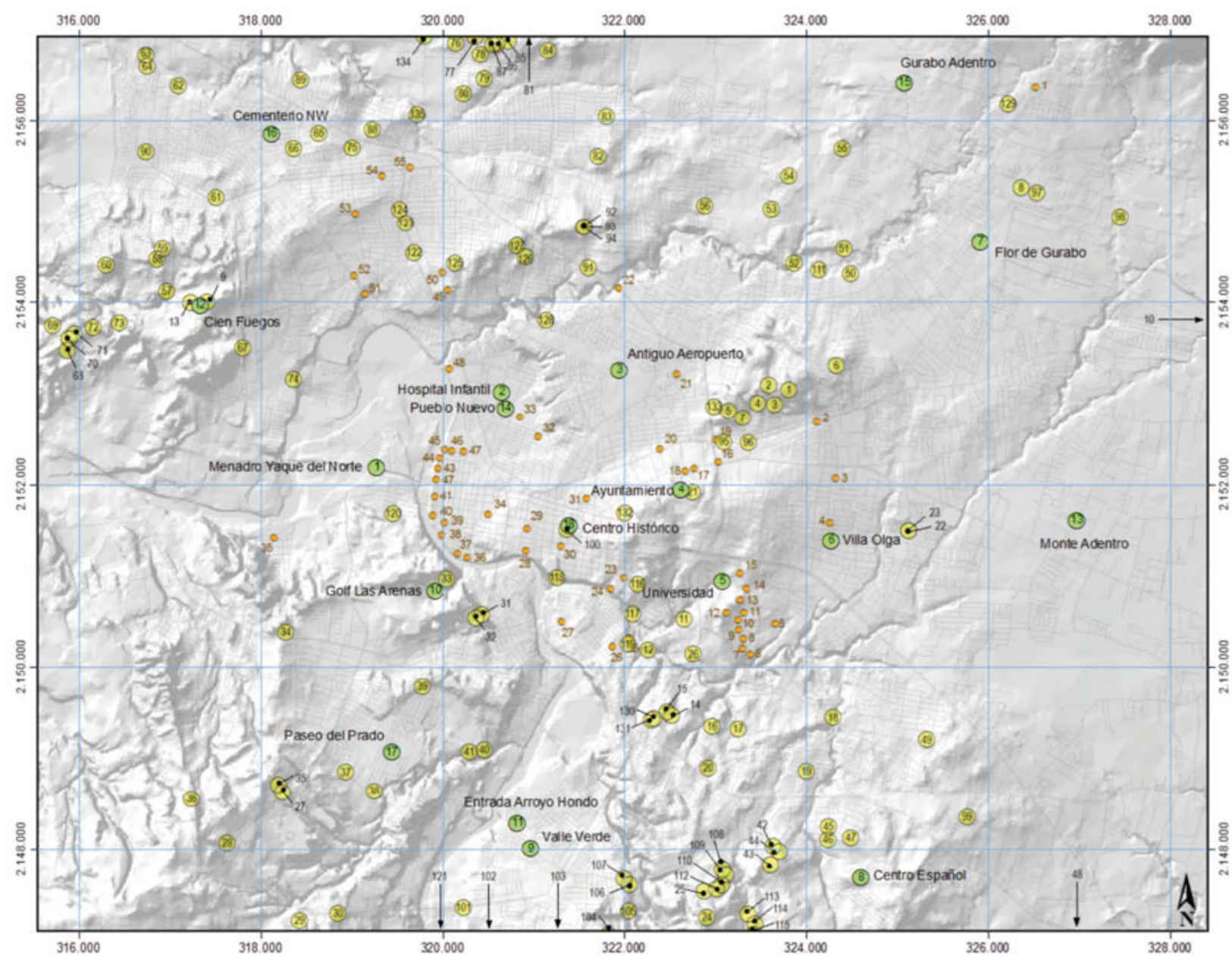

Figura 5. Mapa de sondeos realizados (en verde, con nombre relacionado con el topónimo y número), sondeos recopilados (en marrón) y afloramientos observados (en amarillo y número en negro, las flechas indican afloramientos fuera del marco).

Figure 5. Observations map: geotechnical survey sites in green with name including the place name and number; geotechnical survey sites collected in brown colour; and observed outcrops in yellow and number in black. Arrows indicate outcrops outside the framework.

so en distancias muy cortas. De ese modo, en una misma ciudad, es necesario esperar que los efectos de sitio induzcan de un barrio a otro, variaciones importantes del movimiento vibratorio. Un estudio de microzonificación sísmica consiste en cartografiar las zonas de respuesta sísmica homogénea y proponer movimientos sísmicos de referencia en cada una de estas zonas. Para ello se ha caracterizado el territorio mediante la realización de una campaña geofísica apoyada por los datos geológicos y geotécnicos obtenidos en las fases previas.

\section{Columnas de suelo}

Un análisis conjunto de los datos geológicos, geotécnicos y geofísicos, permite la caracterización de las formaciones superficiales, y la determinación de los parámetros geomecánicos necesarios para los estudios de sitio litológicos. Este análisis nos permite considerar 10 zonas tipo para el estudio de los efectos de sitio en la ciudad de Santiago de los Caballeros. Cada una de las 10 zonas consideradas ha sido definida por una o varias columnas de suelo representativas (Roullé et al., 2010).

Las columnas de suelo representativas del terreno se describen por la litología de los horizontes (suelos blandos, suelos rígidos) la estratigrafía (secuencia y potencia) y las características geomecánicas de los horizontes (en valores medios). La velocidad de ondas $P$, la velocidad de ondas $S$, el peso específico $\rho$ y las curvas de comportamiento dinámico del suelo (rigidez respecto a amortiguamiento respecto a la deformación angular $(\gamma) ; G(\gamma) / G \max$ vs $D(\gamma))$ son 


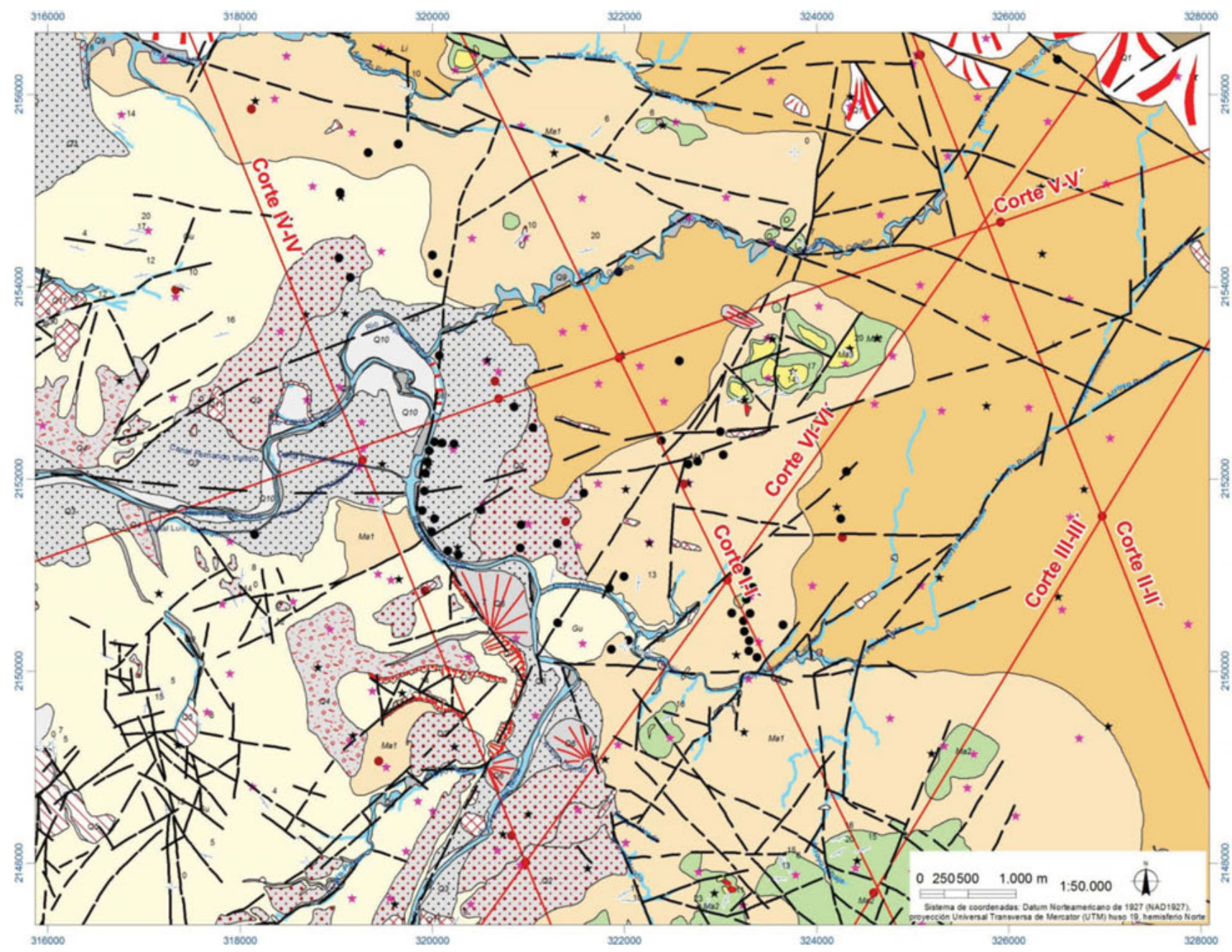

Figura 6. Mapa Geológico de Santiago de los Caballeros. Ver leyenda en Figura 7.

Figure 6. Geological map of Santiago de los Caballeros. Legend in Figure 7.

características dinámicas de los suelos, necesarias en la simulación del comportamiento de una columna de suelo bajo solicitación sísmica.

Cuando las profundidades del sustrato sísmico son inciertas, se pueden calibrar con las medidas $\mathrm{H} / \mathrm{V}$ realizadas sobre el terreno. El método de razon empírica $\mathrm{H} / \mathrm{V}$ del ruido ambiental, o método Nakamura, permite evaluar la frecuencia de resonancia del suelo (Nakamura, 1989). Además de precisar los espesores de las formaciones cartografiadas, otro método geofísico SASW (Spectral Analysis of Surface Waves) permite definir puntualmente un perfil de velocidad de la onda de corte $V_{s}$. De hecho, los espesores de las formaciones geológicas y las velocidades de las ondas de cizallamiento constituyen dos parámetros indispensables para la simulación numérica de la respuesta sísmica de columnas de suelo. Se han ejecutado un total de 147 medidas puntuales $\mathrm{H} / \mathrm{V}$ y 56 perfiles SASW en la zona de estudio.

Se ha simulado el comportamiento del suelo siguiendo un modelo lineal equivalente implementado en CyberQuake (Modaressi et al. 1997). En las simulaciónes numéricas $1 \mathrm{D}$ en CyberQuake se combinan el espectro de amenaza en suelo firme, definido a partir del estudio de peligro sísmico probabilístico (Figura 4), y las columnas de suelo tipo definidas aquí.

\section{Clases de efectos de sitio litológicos y espectros de respuesta asociados}

Los espectros de respuesta específicos en cada zona fueron después representados en un único gráfico 


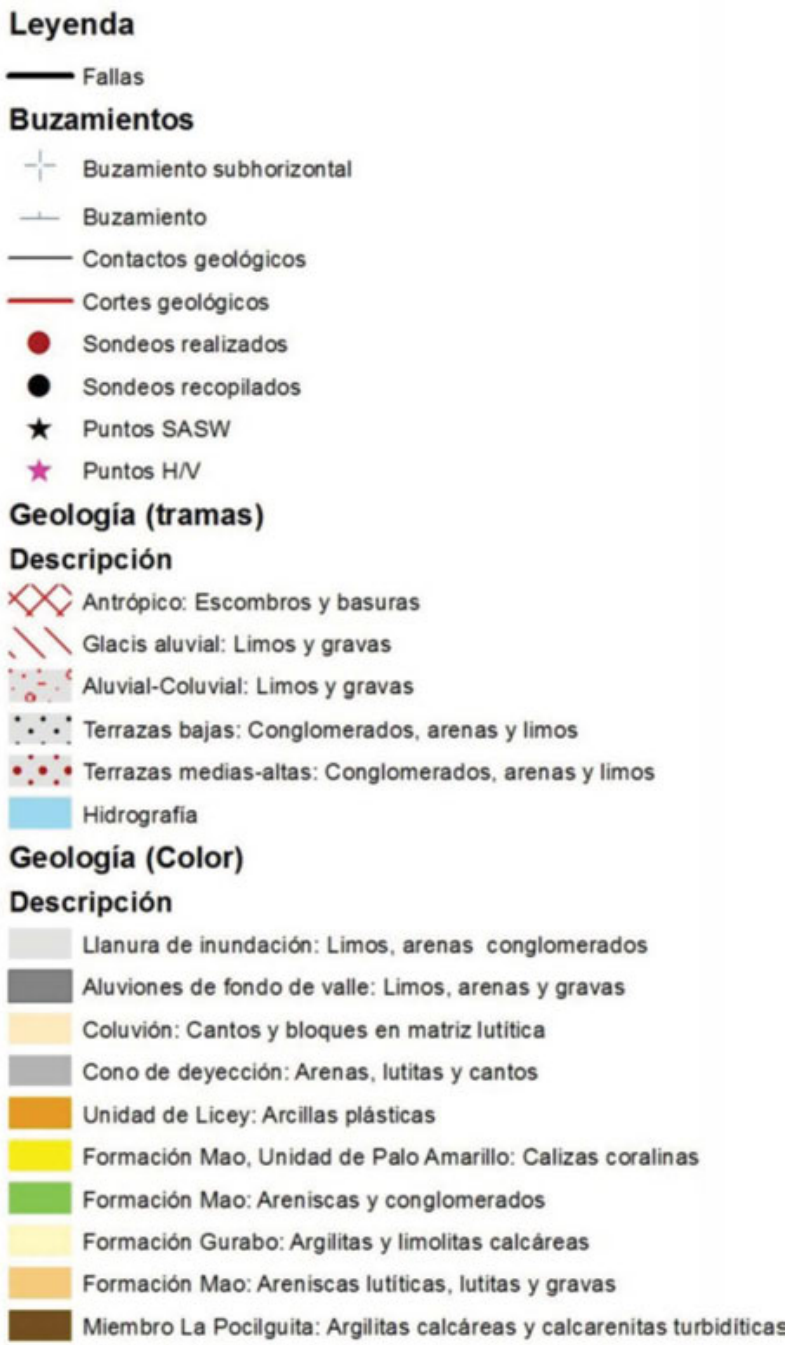

Figura 7. Leyenda del mapa geológico.

Figure 7. Geological map legend.

para evaluar su semejanza y efectuar los agrupamientos de zonas en caso de respuestas sísmicas equivalentes. Esto nos ha permitido definir para toda la zona de estudio 7 clases de efectos de sitio litológicos donde las características de respuesta de los suelos en el caso de sismo serían distintas. Persisten dos sectores, las zonas $n^{\circ} 6$ y $n^{\circ} 8$, donde no se ha podido definir un espectro de respuesta elástica; en estas zonas, los resultados tomados de diferentes métodos son incoherentes el uno con el otro y revelan una fuerte heterogeneidad geométrica y litológica.

La zonificación de clases de suelos a efectos de sitio litológicos y los espectros de respuesta elásticos correspondientes se pueden ver en la Figura 10, en la forma de un mapa de microzonificación sísmica a escala $1 / 25.000$. Su lectura permite conocer en cada punto de la ciudad, las zonas de respuesta sísmica homogénea.

Los resultados indican que el conjunto del territorio de Santiago es susceptible de aumentar las amplificaciones debidas a los efectos de sitio litológicos, en distintos grados y para periodos diferentes. Por ejemplo la clase 0 , al oeste de la ciudad, que corresponde principalmente a la formación Gurabo, manifestaría efectos de sitio que pueden afectar más a los edificios de una o dos plantas. Las terrazas a lo largo del Río Yaque (terrazas bajas y altas terrazas) muestran escasos efectos de sitio pero que podrían afectar principalmente a edificios con dos o cuatro plantas. Las arcillas de la unidad Licey, de gran espesor, es probable que amplifiquen las vibraciones sísmicas para períodos superiores y por tanto que afectan a edificios más altos (de tres a nueve pisos). Ningún lugar de la ciudad puede ser asimilado a un sitio rocoso según la reglamentación sismorresistente nacional (MOPC, 2011).

\section{Licuefacción de suelos}

No existe una metodología general o consensuada para realizar análisis de licuefacción de suelos, pues es un problema complejo. Youd y Perkins (1978) realizaron una clasificación de la susceptibilidad basada en criterios geológicos, mientras que Wakamatsu (1992) incluía condiciones geomorfológicas y genéticas. Otros documentos de referencia de los que se pueden obtener recomendaciones son: Eurocode 8 (1988); AFPS, (1993); TC4-ISSMGE, (1999); CDC-DMG, (1997); y el Chinese Building Code, (1974).

Las recomendaciones en estos documentos tratan de evaluar tanto cualitativa como cuantitativamente, y de forma más o menos detallada, la peligrosidad asociada a la licuefacción. Los trabajos de Seed e Idriss (1971) y Youd e Idriss (1997) son las referencias comunes a todos ellos.

Los factores principales que controlan la susceptibilidad a la licuefacción durante sacudidas sísmicas violentas son: el tipo de depósitos (formación, edad, grado de cementación), las propiedades de los suelos (granulometría, porcentaje de poros, densidad relativa, módulo de corte dinámico) y la profundidad de los acuíferos.

Los ambientes deposicionales de sedimentos controlan la distribución de tamaño de las partículas y, en parte, la densidad relativa y la distribución estructural de partículas. Las características de tamaño de grano de un suelo influyen en su susceptibilidad a la licuefacción. La arena fina tiende a ser más susceptible que limos y gravas. Todo suelo sin cohesión, sin 

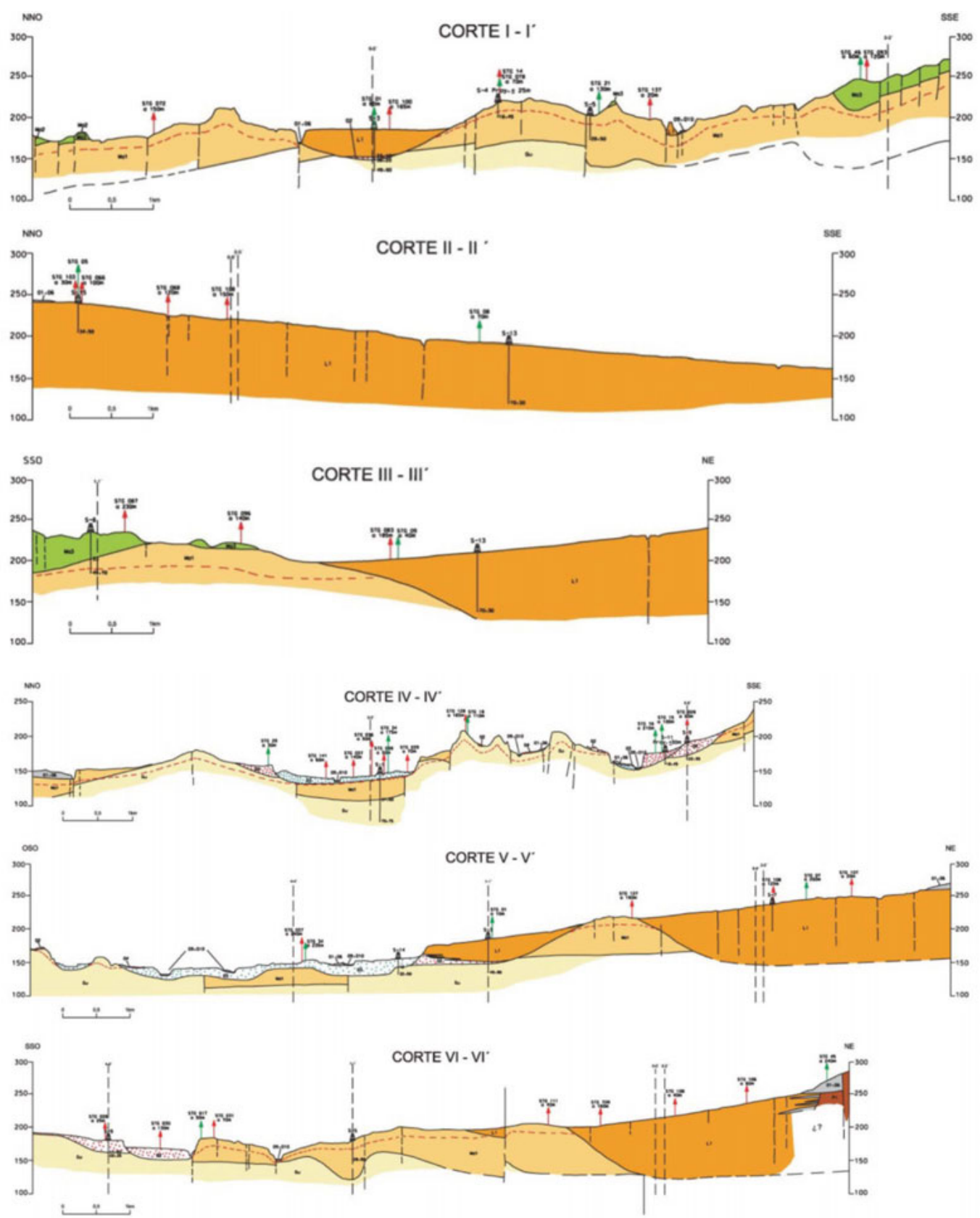

Figura 8. Cortes geológicos. Ver localización en la Figura 5 y leyenda en la Figura 9.

Figure 8. Geological cross sections. Figure 5 shows their location and Figure 9 their legend.

embargo, puede ser considerado potencialmente líquido cuando la influencia de la distribución del tamaño de grano no se considera. En general, los suelos cohesivos que contienen aproximadamente más del $20 \%$ de arcilla pueden ser considerados no licuefactables.

En general, se cree que las características del suelo y los procesos que causan una susceptibilidad menor a fenómenos de licuefacción también causan mayor resistencia de penetración. Por lo tanto, evaluar la resistencia de penetración de una muestra de suelo en un sondeo, es un indicador útil de susceptibilidad a la licuefacción. En el trabajo se realizaron SPTs en suelos a razón de uno cada 1,5 metros hasta los $40 \mathrm{~m}$ y por debajo uno cada $3 \mathrm{~m}$.

Como conclusión se puede resumir que las zonas 


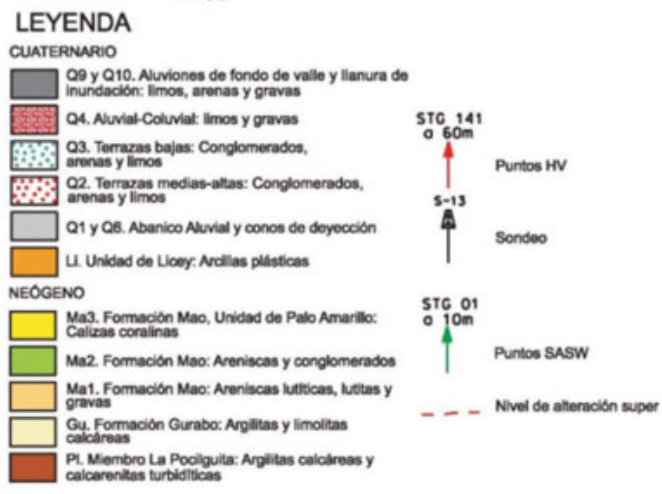

Figura 9. Leyenda de los cortes geológicos. Figure 9. Geological cross-sections legend. donde existen terrenos que son susceptibles a sufrir fenómenos de licuefacción corresponden a:

Zonas donde aparecen materiales granulares sueltos y uniformes tales como depósitos fluviales, coluviales y eólicos así como depósitos antrópicos. Comúnmente corresponden a sedimentos recientes Plio-cuaternarios, abanicos aluviales, depósitos de playa y dunas y otros depósitos de semi-gravedad.

Zonas en las que la profundidad del nivel freático está cerca de la superficie, marismas, puertos, antiguos valles inundados y cauces actuales de los ríos.

Por otro lado, algunos estudios de paleo-licuefacción indican presencia de estructuras de licuefacción asociados a terremotos antiguos a lo largo del valle

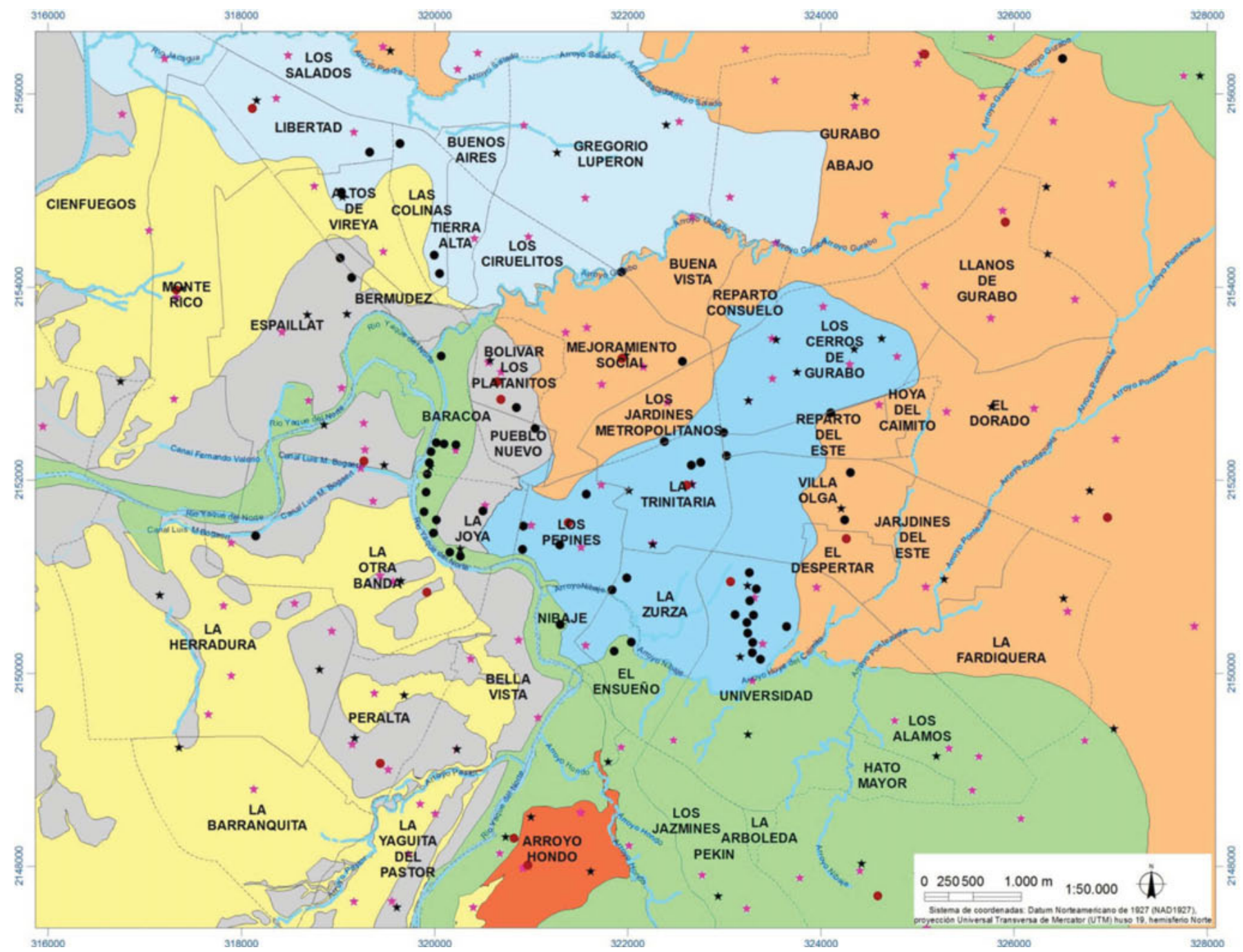

Figura 10. Zonificación de las diferentes clases de efectos de sitio litológicos para la ciudad de Santiago de los Caballeros. Leyenda en la Figura 11.

Figure 10. Site effects zonation of Santiago de los Caballeros. Legend in Figure 11. 


\section{Leyenda}

\begin{tabular}{|c|c|}
\hline 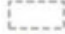 & Barrios \\
\hline - & Sondeos realizados \\
\hline & Sondeos recopilados \\
\hline$\star$ & Puntos SASW \\
\hline$\star$ & Puntos $\mathrm{H} / \mathrm{N}$ \\
\hline & Hidrografia \\
\hline Clase & \\
\hline & Classe 0 \\
\hline & Classe 1 \\
\hline & Classe 2 \\
\hline & Classe 3 \\
\hline & Classe 4 \\
\hline & Casa particular de la zona $\Theta$ \\
\hline & Casa particular de le \\
\hline
\end{tabular}

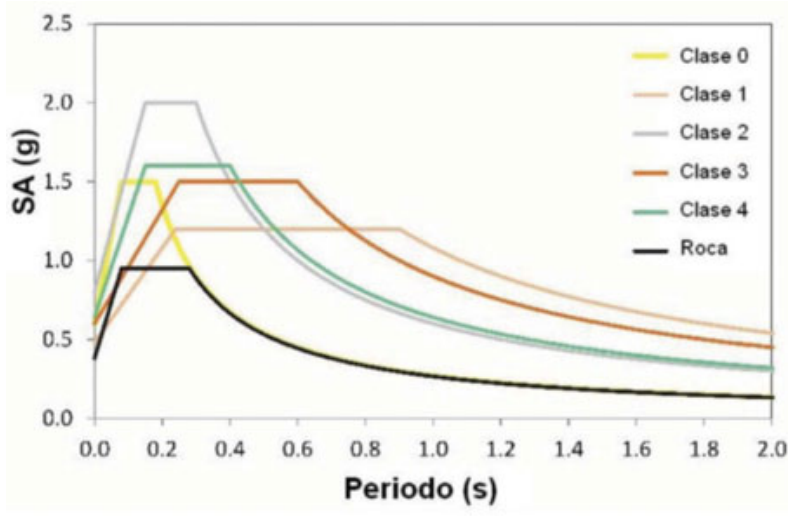

Figura 11. Leyenda del mapa de amplificación sísmica.

Figure 11. Legend of the seismic amplification map.

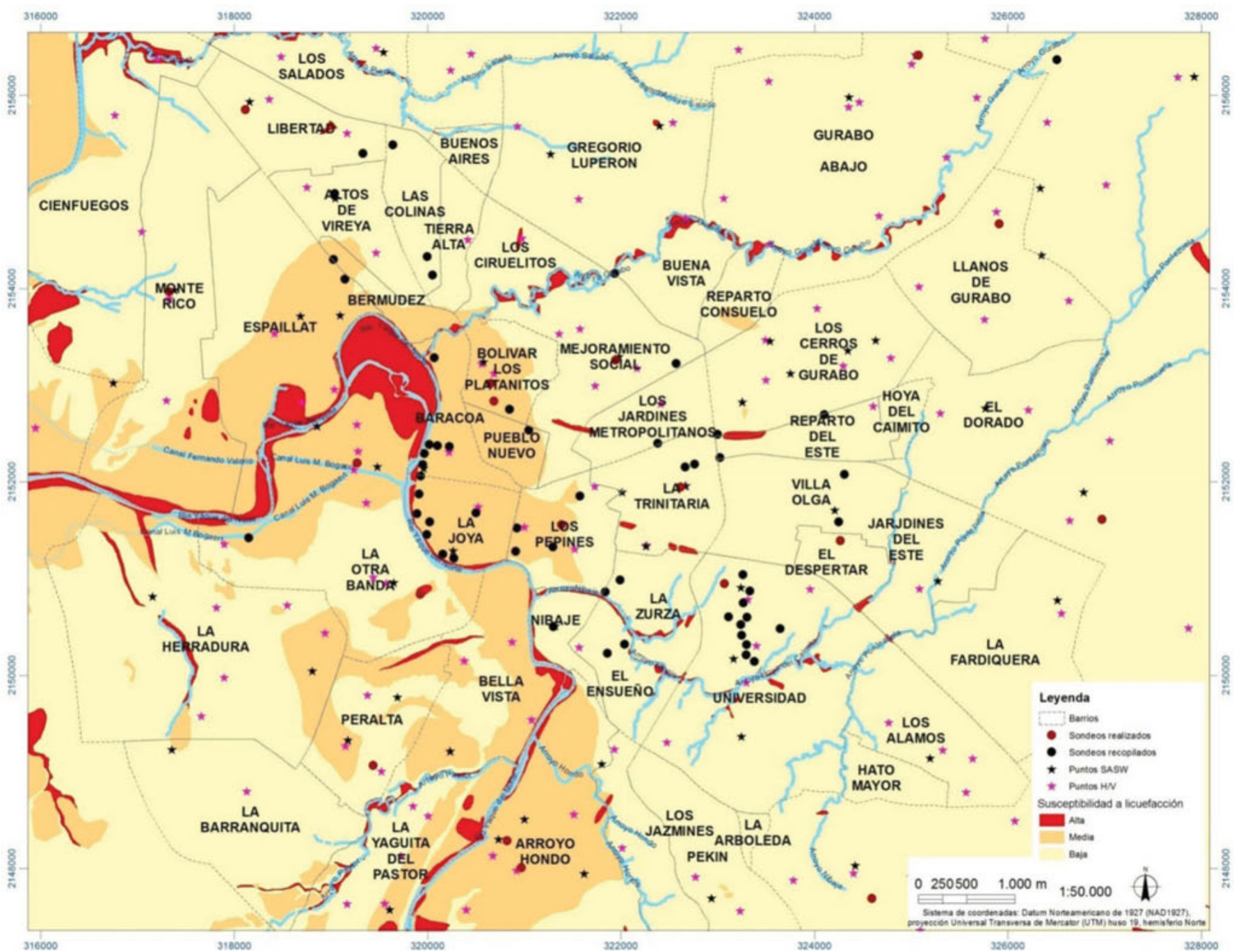

Figura 12. Mapa de susceptibilidad a la licuefacción de Santiago de los Caballeros, República Dominicana.

Figure 12. Map of the liquefaction susceptibility for Santiago de los Caballeros, Dominican Republic. 
Llorente Isidro, M., et al., 2017. Geología para el estudio de microzonación sísmica en... Boletín Geológico y Minero, 128 (3): $715-736$

\begin{tabular}{|c|c|}
\hline Unidad & Susceptibilidad \\
\hline Cordillera Septentrional & Nula o baja \\
\hline Fm. Gurabo & Nula o baja \\
\hline Fm. Mao & Media baja \\
\hline Conos de deyección & Media \\
\hline Terrazas medias-altas & Media \\
\hline Terrazas bajas & Alta \\
\hline Llanura de inundación y canales abandonados & Alta \\
\hline Aluviones de fondo de valle & Media \\
\hline Poligénicos de tipo aluvial-coluvial, glacis aluvial y coluvión & Nula o baja \\
\hline Abanicos aluviales y Unidad Licey & Alta \\
\hline Antrópicos & \\
\hline
\end{tabular}

Tabla 2. Clasificación de las unidades geológicas en función de su grado de susceptibilidad a la licuefacción.

Table 2. Classification of the geological units based on their liquefaction susceptibility.

del Cibao Oriental (Tuttle et al., 2003). El fenómeno de licuefacción ha sido observado en algunos materiales de la ciudad, y es por lo tanto plausible pensar que un futuro terremoto pueda volver a inducir licuefacción en materiales depositados más recientemente y poco compactados.

Atendiendo a todas estas premisas y de acuerdo a los datos de los que se dispone, el análisis de la susceptibilidad cualitativa a la licuefacción permite distinguir 3 niveles de susceptibilidad (alta, media y baja o nula). La susceptibilidad cualitativa así expresada, debe entenderse como una probabilidad relativa de que en una determinada región tenga lugar el fenómeno respecto a otra adyacente. Las regiones o zonas, se agrupan por sus características geológicas, litológicas y geotécnicas considerando las recomendaciones de las diversas fuentes citadas.

La clasificación de las unidades geológicas en función de su grado de susceptibilidad a la licuefacción se puede ver en la Tabla 2 y su representación cartográfica en la Figura 12.

Se ha podido observar que la mayor parte de la ciudad es poco susceptible al fenómeno. Las zonas de mayor susceptibilidad se asocian con los numerosos rellenos antrópicos y depósitos de fondo de barranco en las márgenes de los afluentes del río Yaque del Norte y en las márgenes del propio Yaque del Norte. Muchos de los puntos identificados como de alta susceptibilidad vienen a coincidir con asentamientos urbanos marginales, regiones que son ocupadas por infra-viviendas, un aspecto que es importante tener en cuenta a la hora de planificar actuaciones futuras.

\section{Conclusiones}

Gracias a este trabajo se ha mejorado el conocimiento de la geología superficial en la ciudad de Santiago de los Caballeros. Además, en caso de terremoto en la falla del norte, se ha podido observar que el papel de la geología es fundamental para entender los fenómenos de amplificación de las vibraciones sísmicas, así como de la licuefacción del suelo. Concretamente, el periodo en el que se alcanza la mayor aceleración del suelo para los distintos materiales de Santiago de los Caballeros varía entre los 0,1 a 0,6 s, lo que representa periodos en general mayores que en el caso de roca, con un máximo de amplificación sísmica $x 2$ respecto a los valores en roca (o por efecto exclusivo de la atenuación por distancia). Esto viene a poner de relieve que la amplificación sísmica es muy significativa en la ciudad, concentrándose los valores más altos de aceleración en las zonas situadas más al oeste y al sur y a aquellas ligadas a los depósitos aluviales (Belvaux et al., 2011). Es precisamente hacia el sector oeste hacia donde evoluciona la ciudad y los espectros de aceleración obtenidos por este trabajo deberían tenerse en cuenta a la hora de planificar nuevas edificaciones e infraestructuras.

Por último conviene incidir que en el centro histórico se ha observado mayor incertidumbre en las medidas geofísicas y por consiguiente en los resultados de amplificación sísmica por efecto de sitio. Esta mayor incertidumbre responde a una configuración geológica propia de abanicos coalescentes, con rápidas transiciones de facies, añadido a una importante 
urbanización que dificulta la interpretación. En esta zona (marcada como caso especial de zona 8, Figura 10) sería conveniente realizar nuevos ensayos para minimizar los efectos de incertidumbre mencionados.

\section{Referencias}

AFPS. 1993. Guide méthodologique pour la réalisation d'études de microzonage sismique. Association Française du Génie Parasismique, Francia. 18 pp.

Ali, S.T., Freed, A.M., Calais, E., Manaker, D.M. y McCann, W.R. 2008. Coulomb stress evolution in Northeastern Caribbean over the past 250 years due to coseismic, postseismic and interseismic deformation. Geophysical Journal International, 174, 904-918.

Anderson, J.G. y Luco J.E. 1983. Consequences of slip rate constants on earthquake occurrence relations. Bulletin of the Seismological Society of America, 73, 471-496.

Atkinson G.M. \& Boore D.M. 2003. Empirical groundmotion relations for subduction zone earthquakes and their application to Cascadia and other regions. Bulletin of the Seismological Society of America, 93(4), pp. 17031729.

Becker, J.J. y Sandwell D.T. 2006. SRTM30_PLUS: Data fusion of SRTM land topography with measured and estimated seafloor topography. Scripps Institution of Oceanography, University of California in San Diego, SRTM30 PLUS V2.0.

Belvaux M., Bertil D., Roullé A., Lopera E., Laín-Huerta L., Llorente-Isidro M., Fernández-Merodo J.A., Bernárdez E., Hernaiz Huerta P.P. 2011. Regional and local seismic hazard mapping for seismic risk mitigation of Santiago de los Caballeros (Dominican Republic). 19th Caribbean Geological Conference, Le Gosier, Guadeloupe, French West Indies, 21-24 March 2011, abstract 96, 70-70.

Bernárdez, E. 2004. Mapa geológico de la república Dominicana a escala 1:50.000. Hoja de Mao (5974-I). Proyecto K de Cartografía Geotemática de la República Dominicana. Programa SYSMIN. Dirección General de la Minería. Santo Domingo. 65 pp. 1 mapa 1:50.000.

Bertil D., Lemoine A., Winter T., Belvaux M. 2010. Microzonificación sísmica de Santiago - Republica Dominicana. Amenaza regional. Informe final. Rapport BRGM/RC-59107-FR, $100 \mathrm{p}$.

Boore D. M. and Atkinson G. M. 2008. Ground-motion prediction equations for the average horizontal component of PGA, PGV, and 5\%-damped PSA at spectral periods between $0.01 \mathrm{~s}$ and 10.0s, Earthquake Spectra, 24(1), pp. 99-138.

Calais, E., Mazabraud, Y., Mercier de Lépinay, B., Mann, P., Mattioli, G. y Jansma, P. 2002. Strain partitioning and fault slip rates in the northeastern Caribbean from GPS measurements. Geophysical Research Letters, 29, pp. 1856.

Campbell K. W. \& Bozorgnia Y. 2008. NGA ground motion model for the geometric mean horizontal component of PGA, PGV, PGD and 5\% damped linear elastic response spectra for periods ranging from 0.01 to $10 \mathrm{~s}$, Earthquake Spectra, 24(1), pp. 139-171.
CDC-DMG. 1997. Recommended procedures for implementation of DMG special publication 117 - Guidelines for analyzing and mitigating liquefaction in California. California Department of Conservation, Division of Mines and Geology EEUU.

Chinese Building Code 1974. Earthquake resistant design code for industrial and civils buildings. TJ11-74, China Build.

Chiou B. S.-J. \& Youngs,R. R. 2008. An NGA model for the average horizontal component of peak ground motion and response spectra, Earthquake Spectra, 24(1), pp. 173-215.

Cornell, C. A. 1968. Engineering Seismic Risk Analysis. Bulletin of the Seismological Society of America, 58, pp. 1583-1606.

Darendeli, M.B. 2001. Development of a new family of normalized modulus reduction and material damping curves. Tesis Doctoral, University of Texas, Austin, USA.

DeMets, C., Jansma, P.E., Mattioli, G.S., Dixon, T.H., Farina, F., Bilham, R., Calais, E. y Mann, P. 2000. GPS geodetic constraints on Caribbean-North America plate motion. Geophysical Research Letters, 27, 437-440.

Dolan, J.F. y Wald D.J. 1998. The 1943-1953 north-central Caribbean earthquakes: active tectonic setting, seismic hazards, and implications for Caribbean-North America plate motions. En Dolan, J.F. y Mann P. (eds): Active strike-slip and collisional tectonics of the northern Caribbean plate boundary zone. Geological Society of America, special paper, 326,143-169.

Dolan, J.F., Mullins, H.T. \& Wald D.J. 1998. Active tectonics of the northcentral Caribbean: oblique collision, strain partitioning, and opposing subducted slabs. En Dolan, J.F. y Mann P. (eds): Active strike-slip and collisional tectonics of the northern Caribbean plate boundary zone. Geological Society of America, special paper, 326, 1-62.

Engdahl, E.R. y Villaseñor, A. 2002. Global Seismicity: 19001999. En W.H.K. Lee, H. Kanamori, P.C. Jennings, y C. Kisslinger (eds.): International Handbook of Earthquake and Engineering Seismology. Part A, Chapter 41, pp. 665-690, Academic Press.

Erikson, J.P., Pindell, J.L., Karner, G.D., Sonder, L.J., Fuller, E.Y. y Dent, L. 1998. Neogene Sedimentation and Tectonics in the Cibao Basin and Northern Española: An Example of Basin Evolution near a Strike-SlipDominated Plate Boundary. Journal of Geology, 106, 473-494.

Eurocode 8. 1988. Design provisions for earthquake resistance of structures - Part 1-1: generale rules for the representation of of seismic actions. Part 5: Foundations, retaining structures and geotechnical aspects. CEN. 2004. Eurocode 8: Design of structures for earthquake resistance. Comité Européen de Normalisation (BS EN 1998-1:2004). 232 pp.

Franco O. y Peña, L. 2003. Recopilación - evaluación de los datos geotécnicos-geológicos y reconocimiento geológicos superficial de la zona urbana y peri-urbana norte de Santiago de los Caballeros, para la preparación de mapas de amenazas geológicas sísmicas. Ayuntamiento Municipal de la Ciudad de Santiago de los Caballeros. Informe inédito, $52 \mathrm{pp}$. 
Llorente Isidro, M., et al., 2017. Geología para el estudio de microzonación sísmica en... Boletín Geológico y Minero, 128 (3): $715-736$

Frankel, A., Harmsen, S., Mueller, C., Calais, E. y Haase, J. 2010. Documentation for initial seismic hazard maps for Haiti. U.S. Geological Survey Open-File Report 20101067, $12 \mathrm{p}$.

IGME. 2010. Sondeos geotécnicos para microzonificación sísmica, Santiago de los caballeros, Rep. Dom., CAP2585, GEOCIVIL, Noviembre 2010, 348 pp.

ISC. 2013. International Seismological Centre, On-line Bulletin, http://www.isc.ac.uk, International Seismological Centre, Thatcham, United Kingdom, 2013.

Kelleher, J., Sykes, L. y Oliver, J. 1973. Possible criteria for predicting earthquake locations and their application for major plate boundaries of the Pacific and the Caribbean. Journal of Geophysical Research, 78, pp. 2547-2585.

Kokusho, T. 1980. Cyclic triaxial test of dynamic soil properties for wide strain range. Soils and foundations, 20(4):45-60.

Manaker, D.M., Calais, E., Freed, A.M., Ali, S.T., Przybylski, P., Mattioli, G.S., Jansma, P.E., Prépetit, C. y De Chabalier, J.B. 2008. Interseismic plate coupling and strain partitioning in the Northeastern Caribbean. Geophysical Journal International, 174, pp. 889-903.

Mann, P., Burke, K. y Matumoto, T. 1984. Neotectonics of Hispaniola: plate motion, sedimentation, and seismicity at a restraining bend. Earth and Planetary Science Letters, 70, pp. 311-324.

Mann, P., Calais, E., Ruegg, J.C., DeMets, C., Jansma, P.E. y Mattioli, G.S. 2002. Oblique collision in the northeastern Caribbean from GPS Measurements and geological observations. Tectonics, 21, (6) 1057, doi:10.1029/2001TC001304.

Mann, P., Prentice, C.S., Burr, G., Pea, L.R. y Taylor, F.W. 1998. Tectonic geomorphology and paleoseismology of the Septentrional fault system, Dominican Republic. En: Dolan, J.F. and Mann, P. (eds.), Active Strike-Slip and Collisional Tectonics of the Northern Caribbean Plate Boundary Zone. Geological Society of America Special Paper 326, 63-124.

Mann, P., Taylor, F.W., Lawrence Edwards, R. y Ku, T.L. 1995. Actively evolving microplate formation by oblique collision and sideways motion along strike-slip faults: an example from the northeastern Caribbean plate margin. Tectonophysics, 246, 1-69.

McCann, W.R. 2006. Estimating the threat of tsunamigenic earthquakes and earthquake induced landslide tsunami in the Caribbean. Paper presented at NSF Caribbean Tsunami Workshop, World Scientific, San Juan, Puerto Rico.

McNeill, D. F., Klaus, J.S., Evans. Ch.C. y Budd, A.F. 2008. An Overview of the Regional Geology and Stratigraphy of the Neogene Deposits of the Cibao Valley, Dominican Republic. En: Nehm, R.H. y Budd A.F. (eds): Evolutionary Stasis and Change in the Dominican Republic Neogene. Topics in Geobiology 30, 21-45. Springer.

MIDAS-IPGH. 1997. MIDdle America Seismograph consortium - Instituto Panamericano de Geografía e Historia. In Tanner J.G. and Shepherd, J.B. 1997. Seismic Hazard in Latin America and the Caribean. (1): Project Catalogue and Seismic Hazard Maps, IRDC, Ottawa, 143 pp. http://midas.upr.clu.edu/mds-ipgh.html
Modaressi, H., Foerster, E. y Mellal, A. 1997. Computer aided seismic analysis of soils. Proc. Of the 6th International Symposium On Numerical Models in Geomechanics, NUMOG VI, Montréal, Québec, Canada July 2-4.

MOPC. 2011. Reglamento para el análisis y diseño sísmico de estructuras, Decreto No.201-11, 24 Marzo 2011, Ministerio de Obras Publicas y Comunicaciones, 62 p.

Nakamura Y. 1989. A method for dynamic characteristics estimation of subsurface using microtremor on the ground surface, Quarterly Report of RTRI (Railway Technical Research Institute), 30 (1) 25-32.

NOAA Global Seismicity Catalog CD-ROM, National Centers for Environmental Information, National Oceanic and Atmospheric Administration, https://www.ngdc.noaa. gov/hazard/earthqk.shtml

Penson, E. 1973. El Subsuelo de la Ciudad de Santiago. Primer Congreso Dominicano de Ingenieros, Arquitectos y Agrimensores, CODIA, 102 pp.

Prentice, C.S., Mann, P., Pea, L.R. y Burr, G. 2003. Slip rate and earthquake recurrence along the central Septentrional fault, North American-Caribbean plate boundary, Dominican Republic. Journal of Geophysical Research, 108, doi:10.129/ 2001JB000442.

Prentice, C.S., Mann, P., Taylor, FW., Burr, G. y Valastro, S. 1993. Paleoseismicity of the North American - Caribbean plate boundary (Septentrional fault), Dominican Republic. Geology, 21, 49-52.

Rosencrantz, E. y Mann, P. 1991. SeaMARC II mapping of transform faults in the Cayman Trough, Caribbean Sea. Geology, 19, 690-693.

Roullé A., Vanoudheusden E., Belvaux M., Auclair S. 2011. Microzonificación sísmica de Santiago - Republica Dominicana. Amenaza sísmica local. Informe final. BRGM/RC-59685-FR, 103 p.

Saunders, J.B., Jung, P., y Biju-Duval, B. 1986. Neogene paleontology in the northern Dominican Republic, 1, Field surveys, lithology, environment, and age. Bulletins of American Paleontology, 89 (323), 1-79.

Seed, H.B. e Idriss I.M. 1971. Simplified procedures for evaluating soil liquefaction potential. Journal of the Soil Mechanics and Foundations Division, American Society of Civil Engineers (ASCE) 97, (SM9) 1249-1273.

TC4-ISSMGE. 1999. Manual for Zonation on Seismic Geotechnical Hazard. Revised edition, Technical Committee for Earthquake Geotechnical Engineering (TC4) of the International Society of Soil Mechanics and Geotechnical Engineering. Japón.

Tuttle, M.P., Prentice, C.S., Dyer-Williams, K, Pefra, L.R. y Burr G. 2003. Late Holocene Liquefaction Features in the Dominican Republic: A Powerful Tool for Earthquake Hazard Assessment in the Northeastern Caribbean. Bulletin of the Seismological Society of America, 93, (I) 27-46.

Urien, P. 2010. Mapa Geológico de la República Dominicana escala 1: 50.000, Hoja de Santiago de Los Caballeros (6074-II). Proyecto SYSMIN II. Dirección General de la Minería. 200 pp., 1 mapa 1:50.000.

Wakamatsu, K. 1992. Evaluation of Liquefaction Susceptibility based on Detailed Geomorphlogical Classification. Proceedings, Technical Papers of Annual 
Meeting Architectural Institute of Japan, Vol.B, 14431444.

Weichert, D. 1980. Estimation of the earthquake recurrence parameters for unequal observation periods for different magnitudes. Bulletin of the Seismological Society of America, 70, 1337-1347.

Wells, D.L. y Coppersmith K.J. 1994. New empirical relationships among magnitude, rupture length, rupture width, rupture area and surface displacement. Bulletin of the Seismological Society of America, 84, 974-1002.

Youd, T.L. e Idriss, I.M. (Eds). 1997. Proceedings of the NCEER Workshop on Evaluation of Liquefaction Resistance of Soils. Salt Lake City, UT, January 5-6, 1996, NCEER Technical Report NCEER-97-0022, Buffalo, N.Y.

Youd, T.L. y Perkins, D.M. 1978. Mapping of Liquefaction Induced Ground Failure Potential. Journal of the
Geotechnical Engineering Division, ASCE, Vol.104, No.4, 433-446.

Youd, T.L. y Perkins, D.M. 1987. Mapping of Liquefaction Severity Index. Journal of Geotechnical Engineering Division. 113(11): 1374-1392.

Youngs R. R., Chiou S.-J., Silva W. J. \& Humphrey J. R. .1997. Strong ground motion attenuation relationships for subduction zone earthquakes. Seismological Research Letters, 68(1), 58-73.

Zhao J. X., Zhang J., Asano A., Ohno Y., Oouchi T., Takahashi T., Ogawa H., Irikura K., Thio H. K., Somerville P. G. \& Fukushima Y. 2006. Attenuation relations of strong ground motion in Japan using site classification based on predominant period. Bulletin of the Seismological Society of America, 96(3), 898-913.

Recibido: abril 2015

Revisado: julio 2015

Aceptado: agosto 2015

Publicado: septiembre 2017 